\title{
Radioactively Driven Colloids: \\ A Special Case of Anomalous Diffusion
}

\author{
Graham Wilson, ${ }^{1}$ Amir A. Bahadori, ${ }^{1,}$ a) and Hitesh Bindra ${ }^{1, b)}$ \\ Department of Mechanical and Nuclear Engineering \\ Kansas State University, Manhattan, KS, 66506, USA \\ (Dated: 23 August 2019)
}

A novel concept of self-propelled, radioactively driven colloids is introduced. The focus of this paper is on assessing the impact of alpha emissions on colloidal kinematics. Using Langevin dynamics and a random walk model, a theory has been developed to describe this motion. This theory shows a special case of anomalous diffusion. Numerical simulations have substantiated the theory. It is shown that alpha-particle emission can significantly affect the motion of colloidal particles, although a very short-lived radioisotope is required.

\section{INTRODUCTION}

In the summer of 1827 , the botanist and microscopist Robert Brown observed the peculiar "rapid oscillatory motion" of pollen grains and proved that the particles were not animated by repeating his experiments with inorganic materials ${ }^{1}$. This new-found "Brownian motion" was passive, and did not require any special circumstances to occur. In recent decades, active forms of Brownian motion have been investigated. Particles have been constructed such that when exposed to a gradient electric, temperature, or concentration field, particle motion is induced ${ }^{2,3}$. Just like air- and watercraft require a medium to propel themselves, so too do these phoretic particles. A microscopic analog to spacecraft that exhibits self-propulsion with or without the presence of a medium is a radiation emitting micro/nanoparticle. In this paper, alpha-particle emission is considered, but the analysis can be easily applied to any type of radiation. The work described here is motivated by the lack of a fundamental understanding about radiation-induced movement of colloidal particles.

Although the use of alpha-particle ejection has been considered for the propulsion of macroscopic objects ${ }^{4,5}$, and the effect of electromagnetic radiation on hydrodynamics has been studied $^{6,7}$, the effect of radiation emission by a colloidal particle on colloid dynamics has not been considered. This does not mean, however, that radioactive particles are currently unused. For instance, nanoparticles with radioactive constituents that decay via alpha-particle ejection have been developed for use in targeted alpha-particle therapy, which shows promise as a cancer treatment ${ }^{8-10}$. It is important to understand the role radiation emission plays in the movement of colloidal particles to ensure the proper use of alpha-emitting particles in cancer treatments, and to explore possibilities such as extending the settling time of suspensions or enhancing the diffusivity of colloids.

An effective way to quantify the motion of a particle is its mean square displacement (MSD). In his seminal 1905 paper on Brownian motion, Einstein showed that the MSD of a colloidal particle within a dilute dispersion has a linear relationship with time ${ }^{11}$. Since then, more detailed derivations

a)Electronic mail: bahadori@ ksu.edu

b)Electronic mail: hbindra@ksu.edu have been completed, including those taking into account an additional force term and having short-time accuracy ${ }^{12}$. Furthermore, a phenomenon known as anomalous diffusion was discovered in 1926, where due to non-Brownian factors such as turbulence the MSD is a non-linear function of time ${ }^{13}$. For analyzing active motion of colloidal particles, various models have been developed in literature ${ }^{14,15}$. These existing models take into account transport processes such as, negative mobility and chemotactics ${ }^{16-18}$. Another such factor is momentum imparted to a radioactive colloidal particle by discharge of radiation. This study presents the kinematics of colloidal particles due to alpha emissions and shows that the resulting motion is a special case of anomalous diffusion.

The rest of the paper is organized as follows. In Section II the concept of radioactive decay propelling a colloidal particle is discussed. Section III provides the methods, analytical and numerical, of determining the MSD of a radioactive colloidal particle. The results of both methods are compared in Section IV and the numerical results are analyzed in Section V. Final remarks and conclusions are made in Section VI.

\section{CONCEPT}

When a radioisotope undergoes alpha decay, it emits an alpha particle, and the resulting daughter isotope moves in the other direction in accordance with the conservation of momentum. A colloidal particle composed of radioisotopes moves via a momentum transfer from the daughter isotope when one of its constituent nuclei decays. Since the colloidal particle has a much greater mass than the daughter isotope, the movement is smaller in magnitude than that of an unconstrained nucleus. This is illustrated in Fig. 1, where the dowel pin symbol is the center of mass of the colloidal particle. Viscous drag causes the movement to be finite. A radioactive colloidal particle is comprised of many radioactive atoms, resulting in a center of mass motion due to ejection of alpha particles, as represented in Fig. 2.

Neither Fig. 1 nor Fig. 2 exhibit Brownian motion. The alpha-induced motion shown in these two figures would be in addition to Brownian motion. The exponential decay of the conglomeration of radionuclides within the colloidal particle has an ever-changing effect on the motion of the colloidal particle, resulting in a special case of anomalous diffusion. 


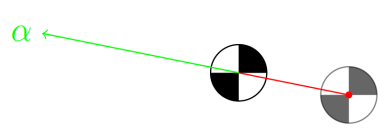

FIG. 1. [Color online] The motion of a colloidal particle due to the ejection of a single alpha particle.

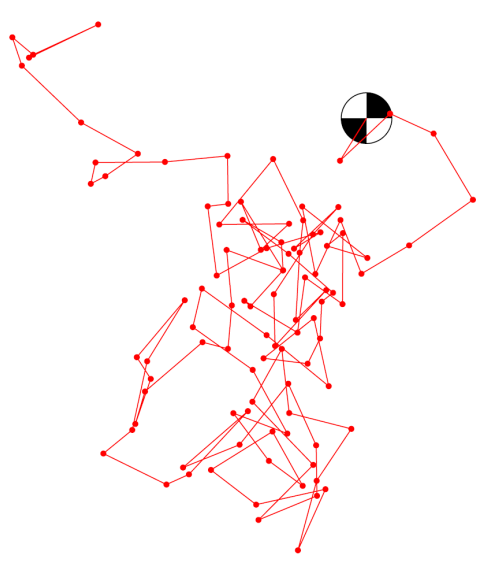

FIG. 2. [Color online] The motion of a colloidal particle due to the ejection of many alpha particles.

Using conservation of momentum, the initial velocity of the colloidal particle due to the emission of an alpha particle is on the order of $10^{-5} \mathrm{~m} / \mathrm{s}$ for the cases considered herein. This results in a Reynolds number on the order of $10^{-5}$; therefore, it is valid to analyze the problem as a Stokes flow.

\section{THEORY}

In Langevin dynamics, particle motion is described with

$$
m \frac{d^{2} \boldsymbol{x}}{d t^{2}}+\gamma \frac{d \boldsymbol{x}}{d t}=\boldsymbol{f}(t),
$$

where $m$ is the mass of the particle, $t$ is time, $\boldsymbol{x}$ is the centerof-mass position vector, $\gamma$ is the drag coefficient, and due to the equipartition theorem $\boldsymbol{f}(t)$ is a Gaussian white noise fluctuating force such that

$$
\begin{aligned}
& \left\langle f_{i}(t)\right\rangle=0, \\
& \left\langle f_{i}(t) f_{j}(t+\tau)\right\rangle=2 \gamma k_{B} T \delta_{i j} \delta(\tau),
\end{aligned}
$$

where $k_{B}$ is the Boltzmann constant and $T$ is the temperature $^{19}$. For a liquid, the effective mass of the par- ticle is $m=m_{p}+(1 / 2) m_{f}$ where $m_{p}$ is the actual mass of the particle and $m_{f}$ is the mass of the displaced fluid ${ }^{20}$. The drag coefficient is $\gamma=6 \pi \mu a$ where $\mu$ is the dynamic viscosity of the fluid and $a$ is the particle radius, assuming spherical particles ${ }^{19}$.

Rewriting the Langevin equation (Eq. 1) as a stochastic differential equation and including the effect of gravity results in an Ornstein-Uhlenbeck process ${ }^{21,22}$

$$
d \boldsymbol{u}_{t}=-\beta\left(\boldsymbol{u}_{t}-\boldsymbol{\mu}\right) d t+\sigma d \boldsymbol{W}_{t},
$$

where $\boldsymbol{u}_{t}$ is the particle velocity, $\beta=\gamma / m,|\boldsymbol{\mu}|=\mid 2 g\left(\rho_{f}-\right.$ $\left.\rho_{p}\right) a^{2} /(9 \mu) \mid$ is the Stokes velocity of a particle with density $\rho_{p}$ in a fluid with density $\rho_{f}, \sigma=\sqrt{2 \gamma k_{B} T} / m$, and $\boldsymbol{W}_{t}$ is the Wiener process ${ }^{19,23}$.

Including the effect of alpha-particle emission in Eq. 4 results in a modified Ornstein-Uhlenbeck process

$$
d \boldsymbol{u}_{t}=-\beta\left(\boldsymbol{u}_{t}-\boldsymbol{\mu}\right) d t+\frac{\boldsymbol{F}_{t}}{m} d t+\sigma d \boldsymbol{W}_{t}
$$

The product $\boldsymbol{F}_{t} d t$ is the momentum change of (i.e. linear impulse applied to) the colloidal particle due to radiation emission. The magnitude of the momentum change due to the ejection of an alpha particle is

$$
M_{0}=|\Delta p|=\sqrt{\frac{E^{2}+2 E m_{\alpha} c^{2}}{c^{2}}},
$$

where $E$ is the kinetic energy of the alpha particle, $m_{\alpha}$ is the rest mass of an alpha particle, and $c$ is the speed of light ${ }^{24}$.

The colloidal particle here is treated as a point particle so that rotational motion due to Brownian forces and the angular impulse from the ejection of alpha particles can be neglected, as can energy loss of the alpha particle as it travels out of the colloidal particle. The escape of recoiling daughter isotopes from the colloidal particle is ignored as well.

\section{A. Analytical Methods}

Integrating Eq. 4 for velocity results into Eq. 7

$$
\boldsymbol{u}_{t}=\boldsymbol{u}_{0} e^{-\beta t}+\boldsymbol{\mu}\left(1-e^{-\beta t}\right)+\sigma e^{-\beta t} \int_{0}^{t} e^{\beta s} d W_{s}
$$

And the integral $\int_{0}^{t} e^{\beta s} d W_{s}$ can also be represented as Weiner process $\frac{1}{\sqrt{2 \beta}} \boldsymbol{W}\left(e^{2 \beta t}-1\right)$ (for more details, see reference ${ }^{23}$ ). This results into the solution for velocity as

$$
\boldsymbol{u}_{t}=\boldsymbol{u}_{0} e^{-\beta t}+\boldsymbol{\mu}\left(1-e^{-\beta t}\right)+\frac{\sigma}{\sqrt{2 \beta}} e^{-\beta t} \boldsymbol{W}\left(e^{2 \beta t}-1\right) .
$$

Substituting the definition of $\boldsymbol{u}_{t}=\frac{d \boldsymbol{x}_{t}}{d t}$, and integrating with respect to time yields ${ }^{12,23}$ 


$$
\begin{aligned}
\boldsymbol{x}_{t}= & \boldsymbol{x}_{0}+\frac{\boldsymbol{u}_{0}}{\beta}\left(1-e^{-\beta t}\right)+\frac{\boldsymbol{\mu}}{\beta}\left(\beta t-1+e^{-\beta t}\right) \\
& +\boldsymbol{W}\left(\frac{\sigma^{2}}{2 \beta^{3}}\left(-3+4 e^{-\beta t}-e^{-2 \beta t}+2 \beta t\right)\right),
\end{aligned}
$$

where $\boldsymbol{x}_{0}$ and $\boldsymbol{u}_{0}$ are the initial position and velocity of the particle, respectively. Eq. 9 describes only the Brownian component of motion.

The movement of a colloidal particle due solely to the discharge of radiation can be approximated as a random walk on a three-dimensional lattice. The step length is $\varepsilon=M_{0} / \gamma$, or the finite distance traveled by a sphere propelled by an impulse $^{19}$. Successive steps occur at a frequency equal to the activity of the radioisotope $1 / \delta t=A=\lambda N_{0} e^{-\lambda t}$, where $\lambda=\ln (2) / T_{1 / 2}$ is the decay constant of the radionuclide, $T_{1 / 2}$ is the half-life of the radionuclide, $N_{0}=m_{p} N_{A} / \mathscr{A}$ is the number of radioactive nuclei in the colloidal particle at $t=0$, $N_{A}$ is Avogadro's number, and $\mathscr{A}$ is the atomic mass of the radioisotope ${ }^{24}$.

Define $P_{N}(m, n, o)$ as the probability to find the random walker colloidal particle at position $(x, y, z)=(m \varepsilon, n \varepsilon, o \varepsilon)$ at time $t=N \delta t$, where $(m, n, o)$ are integers. Considering an equal probability of motion in all directions, we have

$$
\begin{gathered}
P_{N+1}(m, n, o)=\frac{1}{6} P_{N}(m-1, n, o)+\frac{1}{6} P_{N}(m+1, n, o) \\
+\frac{1}{6} P_{N}(m, n-1, o)+\frac{1}{6} P_{N}(m, n+1, o) \\
+\frac{1}{6} P_{N}(m, n, o-1)+\frac{1}{6} P_{N}(m, n, o+1) .
\end{gathered}
$$

In the limit of large $N$ and small $\varepsilon$

$$
\delta t \frac{\partial P}{\partial t}=\frac{\varepsilon^{2}}{6}\left(\frac{\partial^{2} P}{\partial x^{2}}+\frac{\partial^{2} P}{\partial y^{2}}+\frac{\partial^{2} P}{\partial z^{2}}\right) .
$$

Thus as the step size approaches zero and the frequency approaches infinity, a random walk converges to the diffusion equation

$$
\frac{\partial f}{\partial t}=D \nabla^{2} f, \quad D=\frac{\varepsilon^{2}}{6 \delta t},
$$

where $D$ is the diffusion coefficient and $f$ is the timedependent probability of the particle's location ${ }^{25}$. Since Eq. 12 results in a normal distribution for $f$, the MSD of the particle is given by $\left\langle\left(\boldsymbol{r}(t)-\boldsymbol{r}_{0}\right)^{2}\right\rangle=6 D t$. A random walk may then be described by the Wiener process $W\left(\varepsilon^{2} t /(3 \delta t)\right)$, the variance in one dimension being one third of the total variance.

In the case of a radioactively driven colloid, the diffusion coefficient is dependent on time and thus the diffusion equation is solved with respect to $t^{\prime}$ instead of $t$, where $t^{\prime}=$ $\int_{0}^{t} e^{-\lambda s} d s=\left(1-e^{-\lambda t}\right) / \lambda$. The modified MSD is then given by $6 D_{\text {rad }}^{\prime} t^{\prime}=N_{0}\left(M_{0} / \gamma\right)^{2}\left(1-e^{-\lambda t}\right)$ and the diffusion coefficient is $D_{\text {rad }}=N_{0}\left(M_{0} / \gamma\right)^{2}\left(1-e^{-\lambda t}\right) /(6 t)$. Thus the stochastic process describing the position of the radioactive colloidal particle is

$$
\boldsymbol{x}_{t}=\boldsymbol{x}_{0}+\frac{\boldsymbol{u}_{0}}{\beta}\left(1-e^{-\beta t}\right)+\frac{\boldsymbol{\mu}}{\beta}\left(\beta t-1+e^{-\beta t}\right)+\boldsymbol{W}\left(\frac{\sigma^{2}}{2 \beta^{3}}\left(-3+4 e^{-\beta t}-e^{-2 \beta t}+2 \beta t\right)+\frac{N_{0}}{3}\left(\frac{M_{0}}{\gamma}\right)^{2}\left(1-e^{-\lambda t}\right)\right)
$$

Letting $t$ approach infinity results in $\boldsymbol{x}_{t}=\boldsymbol{\mu} t+(\sigma / \beta) \boldsymbol{W}_{t}$ from which it can be seen that $D_{0}=k_{B} T / \gamma$, the StokesEinstein equation.

This is a special case of anomalous diffusion, where the MSD is essentially only a non-linear function of time from the initialization of the system until a timescale similar to that of the half-life. This aging process is unique in that it does not exhibit a traditional sub- or super-diffusive behavior, but rather transitions from anomalous diffusion to normal diffusion. This exponentially decaying behavior is reminiscent of the transient terms in the Brownian MSD, but with a much greater magnitude and duration.

Due to the central limit theorem and the random walk approximation, Eq. 13 may not be converged to the true colloidal particle position at very short times because not enough steps will have been taken. Furthermore, the discharge of radiation was approximated to occur on a three-dimensional lattice instead of isotropically. These shortcomings suggest that a more detailed model is required.

\section{B. Numerical Methods}

In order to verify the accuracy of the stochastic process describing particle position (Eq. 13), and to provide a more accurate model for short times, the physics of individual alphaparticles were simulated to compute the $\boldsymbol{F}_{t} d t$ term in the Langevin model of Eq. 5. The velocity of the particle was found with Euler-Maruyama (EM) integration, and then the position was found via trapezoidal integration ${ }^{26}$. For stability of the solution, the time step size was chosen such that for a pure Ornstein-Uhlenbeck process the EM approximate solution would be stable in distribution $(\Delta t<1 / \beta)^{27}$.

To apply a numerical method to Eq. 5, the interval of simulation is discretized with time steps $\Delta t_{j}$. Let $\tau_{j}=\sum_{n=1}^{j} \Delta t_{n}$. The numerical approximation to $\boldsymbol{u}\left(\tau_{j}\right)$ is denoted $\boldsymbol{u}_{j}$. The EM method takes the form 


$$
\begin{array}{r}
\boldsymbol{u}_{j}=\boldsymbol{u}_{j-1}-\boldsymbol{\beta}\left(\boldsymbol{u}_{j-1}-\boldsymbol{\mu}\right) \Delta t_{j}+\frac{\Delta \boldsymbol{p}_{j}}{m} \\
+\boldsymbol{\sigma}\left(\boldsymbol{W}\left(\tau_{j}\right)-\boldsymbol{W}\left(\tau_{j-1}\right)\right) .
\end{array}
$$

The escape of recoiling daughter isotopes from the colloidal particle is still neglected, and the colloidal particle is still considered a point particle for rotational purposes, but not for interactions of alpha particles with matter. The $\Delta p_{j}$ term in Eq. 14 is obtained via the following simulation steps:

1. Randomly choose the location $r$ of the alpha decay with a uniform distribution for location within the colloidal particle $^{28}$

2. Randomly choose the radiation emission direction $\Omega$ with a uniform distribution for direction over $4 \pi$ steradians ${ }^{29}$.

3. Calculate the distance $\Delta x$ the alpha particle travels from the decay location until the point where it exits the colloidal particle.

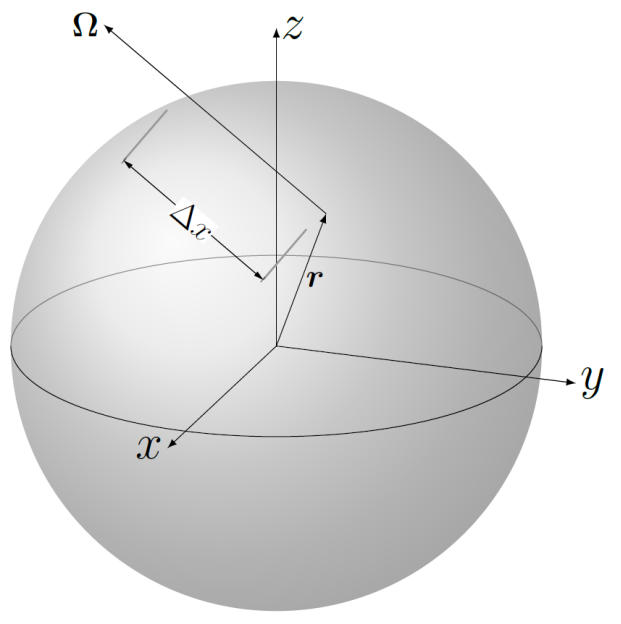

FIG. 3. An example geometry for a simulated alpha decay.

4. Calculate the energy of the alpha particle as it exits the colloidal particle with

$$
E_{\text {exit }}=E_{\alpha}-\left.\frac{d E}{d x}\right|_{t o t} \Delta x,
$$

where $E_{\alpha}$ is the initial energy of the alpha particle and $-d E /\left.d x\right|_{t o t}$ is the stopping power of the colloidal particle. Since only a fraction of the alpha particle's energy would be lost if it traveled through the entire diameter of the colloidal particle, the stopping power is taken to be constant.
5. Use $E_{\text {exit }}$ in Eq. 6 to determine the momentum imparted to the colloidal particle in the direction opposite of the radiation discharge.

6. The net momentum change from all alpha particles emitted in a time step is $\Delta \boldsymbol{p}_{j}$.

The particles simulated had a radius of $0.5 \mu \mathrm{m}$ and were suspended in water at $20^{\circ} \mathrm{C}$ (see Ref. ${ }^{30}$ and Ref. ${ }^{31}$ for the correlations used for the density and viscosity of water, respectively). Particles composed of the four materials listed in Table I were simulated. To understand the effect of radiation discharge, "particle pairs" were simulated such that both particles in a pair had the same random Brownian component of motion, but only one took into account the effect of alphaparticle emission. Example trajectories from one particle pair are plotted in Fig. 4. For accurate statistics 10,000 particle pairs were simulated for each scenario.

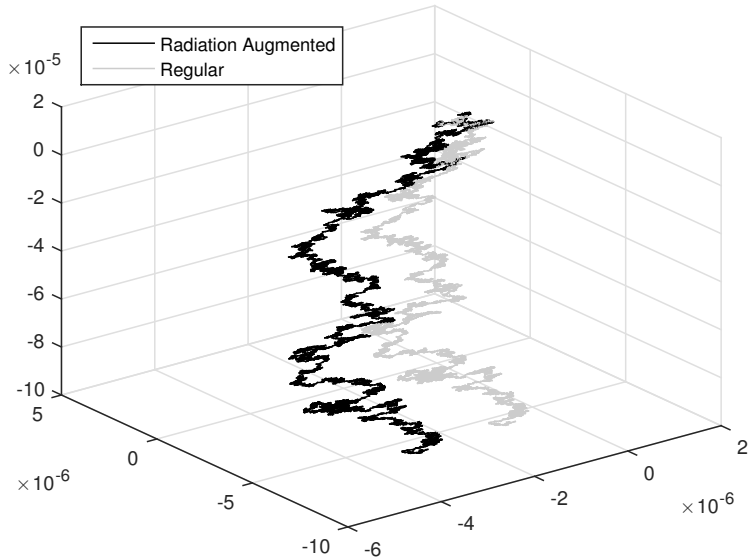

FIG. 4. The trajectories of a particle pair

TABLE I. Alpha-decaying radioisotopes used in the simulations ${ }^{32-36}$.

\begin{tabular}{lcccc}
\hline \hline Case & 1 & $2(\mathrm{a})$ & $2(\mathrm{~b})$ & 3 \\
\hline Isotope & ${ }^{222} \mathrm{Ra}$ & ${ }^{225} \mathrm{~Pa}$ & ${ }^{222} \mathrm{Th}$ & $218 \mathrm{Ac}$ \\
$\rho\left(\mathrm{g} / \mathrm{cm}^{3}\right)$ & 5.0 & 15.37 & 11.72 & 10.07 \\
$T_{1 / 2}(\mathrm{~s})$ & 36.2 & 1.7 & $2.24 \times 10^{-3}$ & $1.1 \times 10^{-6}$ \\
$E_{\alpha}(\mathrm{MeV})$ & 6.556 & 7.22 & 7.98 & 9.20 \\
$\mathscr{A}(\mathrm{g} / \mathrm{mol})$ & 222 & 225 & 222 & 218 \\
$-\left.\frac{d E}{d x}\right|_{t o t}(\mathrm{MeV} / \mathrm{mm})$ & 101.8 & 296.5 & 214.4 & 176.5 \\
\hline \hline
\end{tabular}

In order to determine whether different computational timescales of alpha-particle ejection and Brownian motion would change the computed position of the colloidal particle, simulations of two different time step types were conducted. The first time step was set to the value of $5 \times 10^{-7}$ seconds for ${ }^{222} \mathrm{Th}$ and ${ }^{225} \mathrm{~Pa}$ and $1 \times 10^{-7}$ seconds for ${ }^{222} \mathrm{Ra}$ and ${ }^{218} \mathrm{Ac}$. Both time step values were near the stability limit for each particle to reduce computational expense. In this case the number of nuclei that decayed in each time step was determined 
as a Poisson random variable with a mean of $\int_{t}^{t+\Delta t} A(s) d s=$ $N_{0} e^{-\lambda t}\left(1-e^{-\lambda \Delta t}\right)$. The second time step was adaptive such that on average there would be one decay per time step. The decay constant is defined as $\lambda \equiv \lim _{\Delta t \rightarrow 0}(\Delta N / N) / \Delta t$, so the time step was set to $\Delta t=1 / \lambda N(t)$, where $N(t)$ is the number of undecayed nuclei ${ }^{24}$. In this case the number of nuclei that decayed in each time step was determined as a Poisson random variable with a mean of 1 . Once the adaptive time steps grew larger than the time step size from the former case, the set time step size and corresponding mean number of decays from the former case were implemented.

\section{RESULTS}

For the following plots, the MSD was computed about the displacement due to gravity (from the $\boldsymbol{\mu}$ term in Eq. 13) so that the variance of the Wiener process is more readily observable. The theoretical MSD, $\left\langle\left(\boldsymbol{r}(t)-\boldsymbol{r}_{0}\right)^{2}\right\rangle_{\text {theor }}$, the MSD computed from the numerical simulations, $\left\langle\left(\boldsymbol{r}(t)-\boldsymbol{r}_{0}\right)^{2}\right\rangle$, and the $1 \sigma$ error band about the numerical MSD, $\left\langle\left(\boldsymbol{r}(t)-\boldsymbol{r}_{0}\right)^{2}\right\rangle \pm \sigma$, are all plotted with and without the effect of radioactivity.

In Case 1, there is no significant difference in the motion of a colloidal particle with and without the effect of alphaparticle emission. Case 2 highlights the difference in motion between a radioactive and non-radioactive colloidal particle. In Case 3, the adaptive and set time step simulations give different results.

For Case 1, the MSDs for ${ }^{222} \mathrm{Ra}$ are plotted in Fig. 5, with the numerical MSD from the adaptive time step case. The MSD considering the effect of alpha-particle emission is only slightly larger than the MSD without the effect because the half-life of ${ }^{222} \mathrm{Ra}$ is relatively long. Both the Brownian and radioactive parts of the numerical simulations show good agreement with Eq. 13. The numerical MSD from the set time step case was virtually identical to that from the adaptive case.

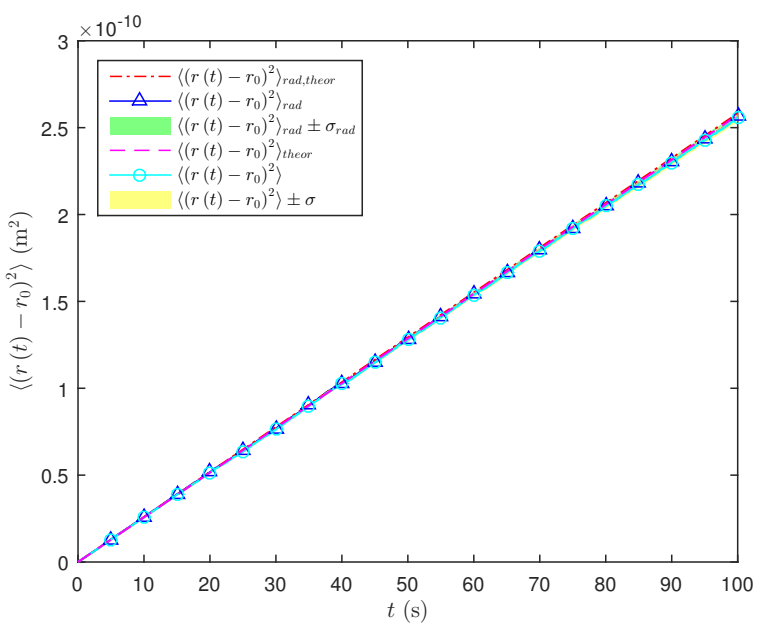

FIG. 5. [Color online] The MSDs for ${ }^{222} \mathrm{Ra}$.

For Case 2(a), the MSDs for ${ }^{225} \mathrm{~Pa}$ are plotted in Fig. 6, with the numerical MSD from the adaptive time step case.
The anomalous diffusion is of an order of magnitude comparable to the regular diffusion. Both the Brownian and radioactive parts of the numerical simulations are in agreement with Eq. 13. At very short times, the MSDs behave as shown in Fig. 7. There is an offset between the theoretical and numerical MSDs when radiation is considered as allowed by the central limit theorem and the random walk approximation as discussed earlier. The Brownian theory and simulation results are still in accord. For the radioactive case, the numerical MSD converges fairly quickly to the theoretical MSD, as seen in Fig. 8.

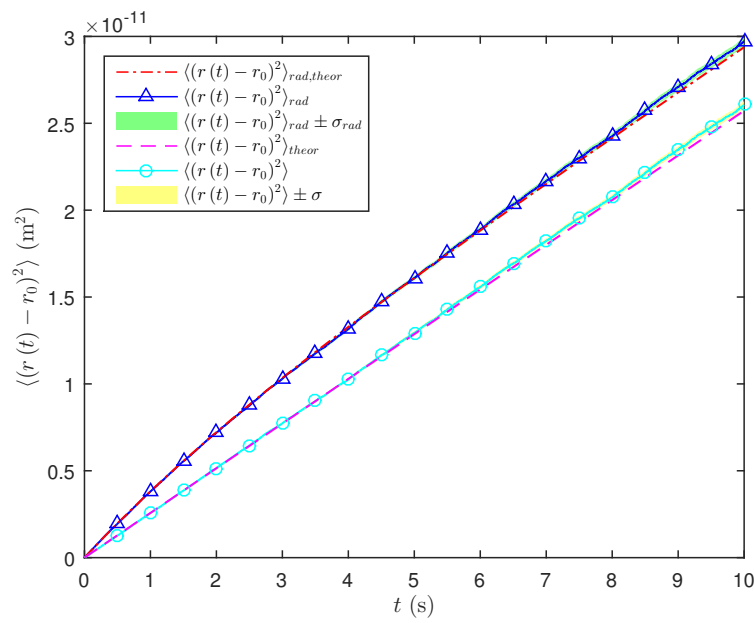

FIG. 6. [Color online] The MSDs for ${ }^{225} \mathrm{~Pa}$.

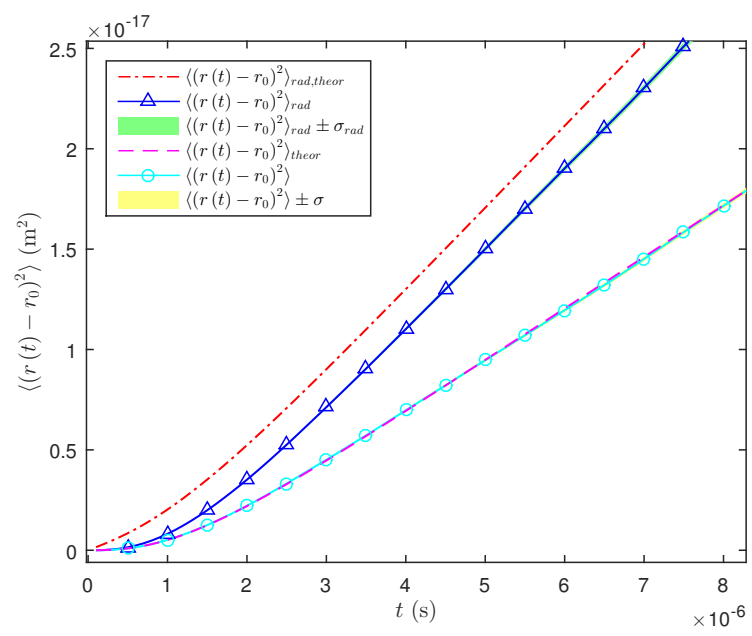

FIG. 7. [Color online] The MSDs for ${ }^{225} \mathrm{~Pa}$ at very short times.

For Case 2(b), the MSDs for ${ }^{222} \mathrm{Th}$ are plotted in Fig. 9, with the numerical MSD from the adaptive time step case. Anomalous diffusion is apparent in this case because of the short half-life of ${ }^{222} \mathrm{Th}$. The Brownian and radioactive theory and simulation results are congruous. For both isotopes in Case 2, the numerical MSDs from the adaptive and set time step cases were practically equal. 


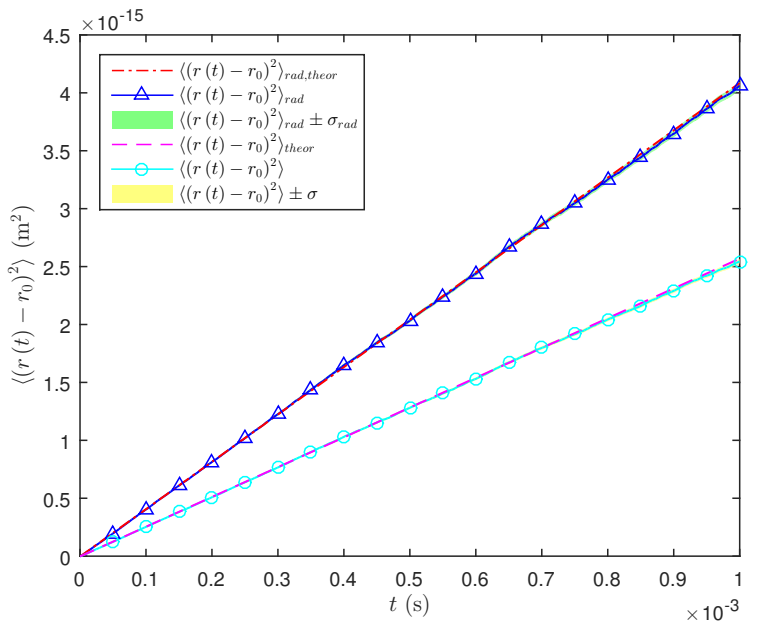

FIG. 8. [Color online] The MSDs for ${ }^{225} \mathrm{~Pa}$ at short times.

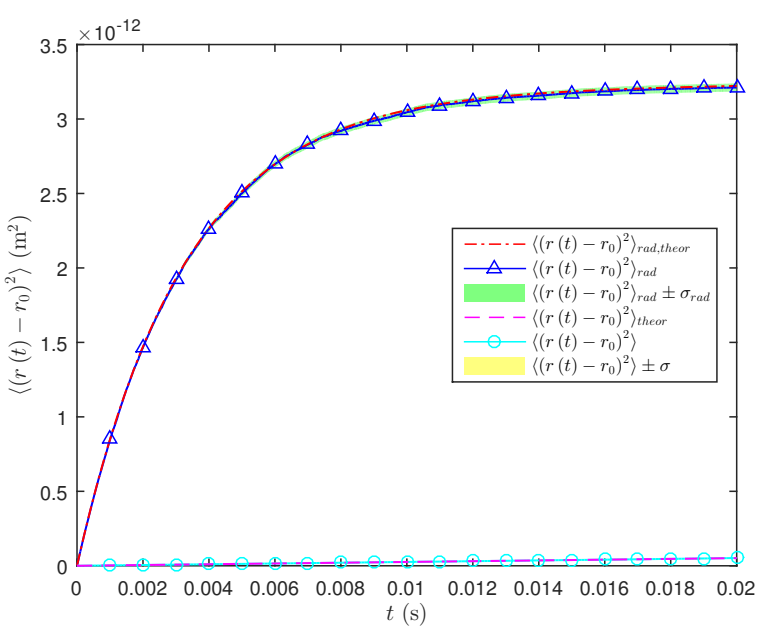

FIG. 9. [Color online] The MSDs for ${ }^{222} \mathrm{Th}$.

For Case 3, the MSDs for ${ }^{218}$ Ac are plotted in Fig. 10 for the case when radiation emission is considered, with the set time step size for the numerical MSD. The theoretical and numerical MSDs never match.

In Fig. 11 the MSDs are plotted for the case when radiation emission is considered, with the adaptive time step size for the numerical MSD. The theoretical and numerical MSDs are not in accord at first, but the simulation results do eventually converge to theory. With such a short half-life, the computational time step size does play a role: the limiting simulation value for the adaptive case shows good agreement with theory, but the limiting simulation value for the set case does not. The offset at earlier times for both cases is allowed by the central limit theorem and is so pronounced for this isotope as compared to the others because of the short half-life. Anomalous diffusion is quite prominent because of the short half-life as well.

As shown in Fig. 12, the Brownian theory and simulation results still match.

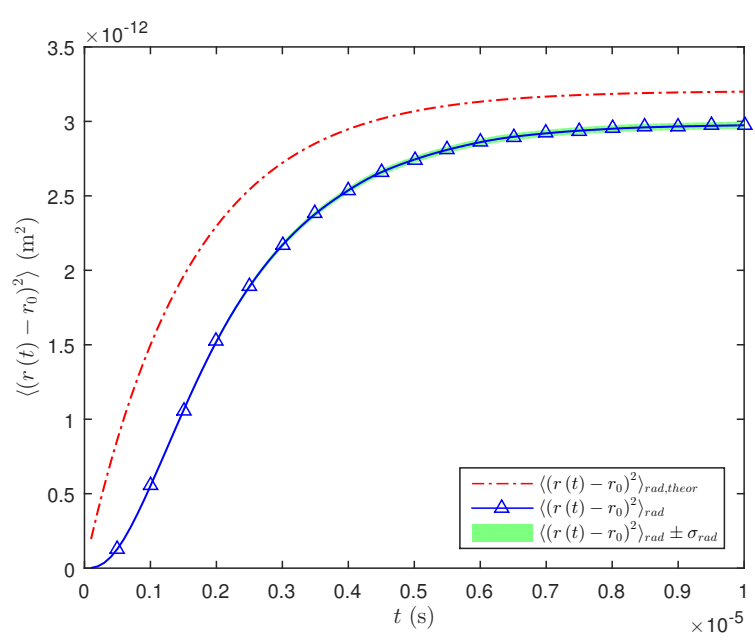

FIG. 10. [Color online] The set time step MSDs for ${ }^{218} \mathrm{Ac}$ for the radioactive case.

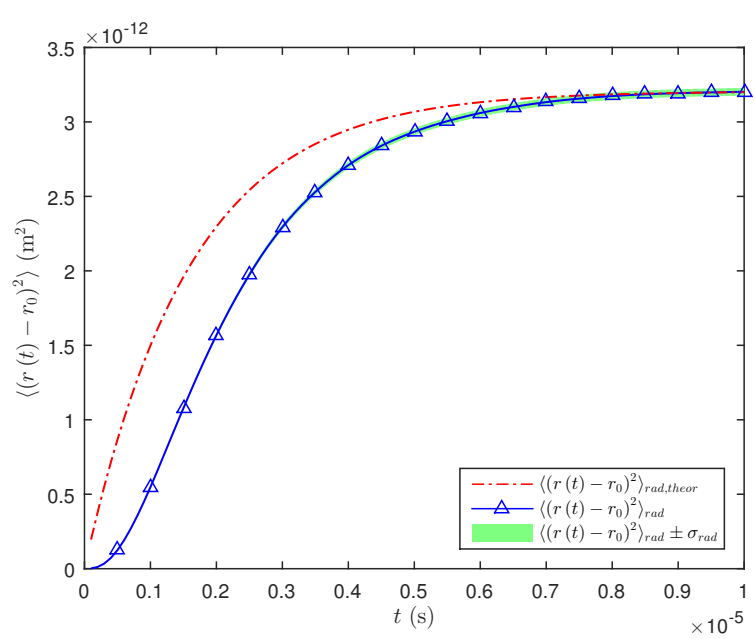

FIG. 11. [Color online] The adaptive time step MSDs for ${ }^{218} \mathrm{Ac}$ for the radioactive case.

\section{ANALYSIS}

To ensure effective random number generation within the numerical simulations, the autocorrelation function of velocity data and the excess kurtosis of the colloidal particle position were both computed. The autocorrelation functions for both the non-radioactive and radioactive adaptive time step cases with ${ }^{225} \mathrm{~Pa}$ are plotted in Fig. 13 and Fig. 14, respectively. Values larger in magnitude than the solid/dashed lines mean that the null hypothesis that there is no autocorrelation at and beyond a given lag is rejected at a significance level of $95 / 99 \%$. Thus, from the figures, the velocity is random with and without consideration of alpha-particle ejection.

The sample kurtosis $b_{2}$ of the three-dimensional colloidal particle position was calculated as in Ref. ${ }^{37}$. The threedimensional kurtosis of a Gaussian population is $\beta_{2}=15$ and the three-dimensional excess kurtosis of a Gaussian popula- 


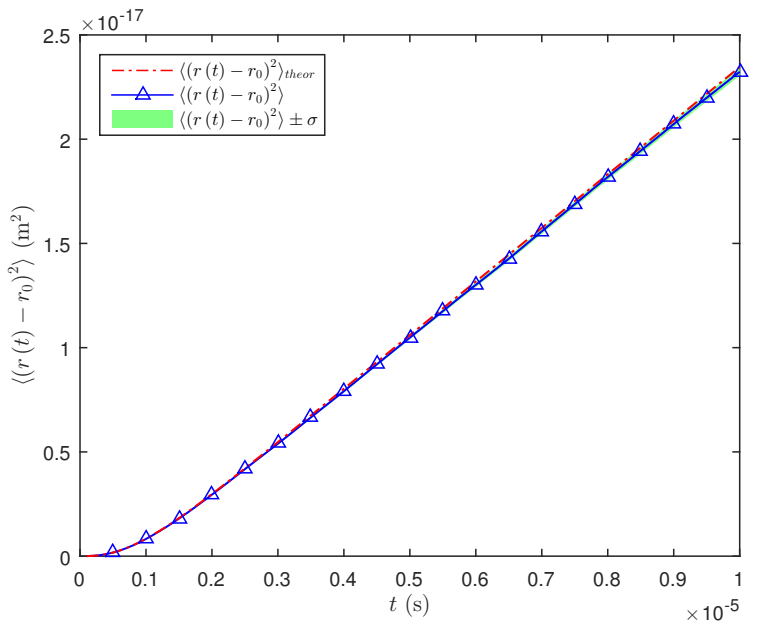

FIG. 12. [Color online] The adaptive time step MSDs for ${ }^{218} \mathrm{Ac}$ for the non-radioactive case.

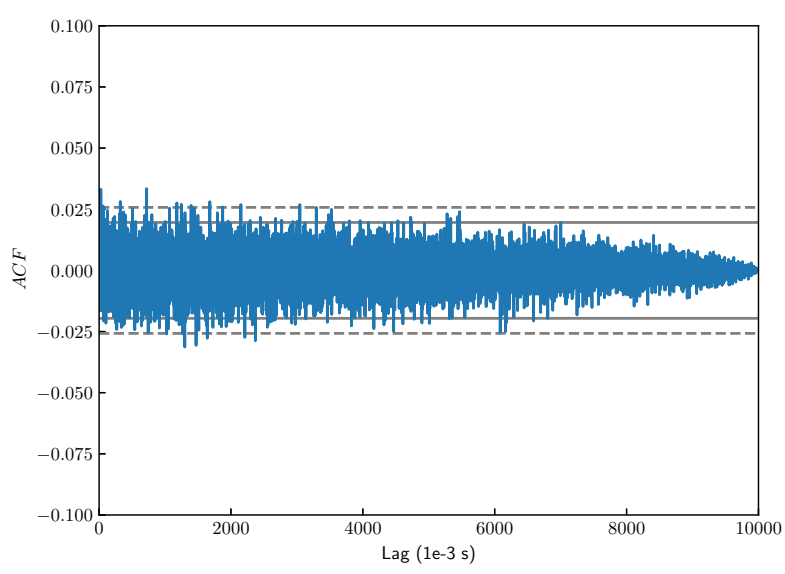

FIG. 13. [Color online] The autocorrelation function of the $u$ velocity data from the non-radioactive, adaptive time step case for ${ }^{225} \mathrm{~Pa}$.

tion is $\gamma_{2}=0$; therefore, the excess sample kurtosis of the three-dimensional colloidal particle position is $g_{2}=b_{2}-15$. In Fig. 15, the excess sample kurtosis of the three-dimensional colloidal particle position about the displacement due to gravity for the adaptive time step case with ${ }^{225} \mathrm{~Pa}$ is plotted. From the figure it can be seen that the particles are approximately normally distributed, as is expected from a Wiener process.

\section{CONCLUSIONS}

A new concept for the propulsion of colloidal particles is presented due to the emission of radiation. Langevin equation based model is devised and the results presented in this study exhibit a special case of anomalous diffusion. It was shown that the effect of alpha-particle ejection on the motion of a colloidal particle can be consequential, is most readily

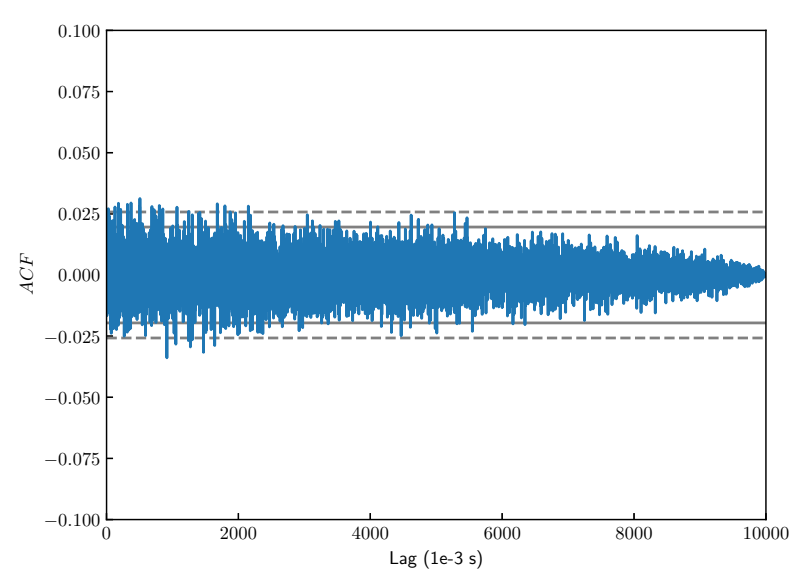

FIG. 14. [Color online] The autocorrelation function of the $u$ velocity data from the radioactive, adaptive time step case for ${ }^{225} \mathrm{~Pa}$.

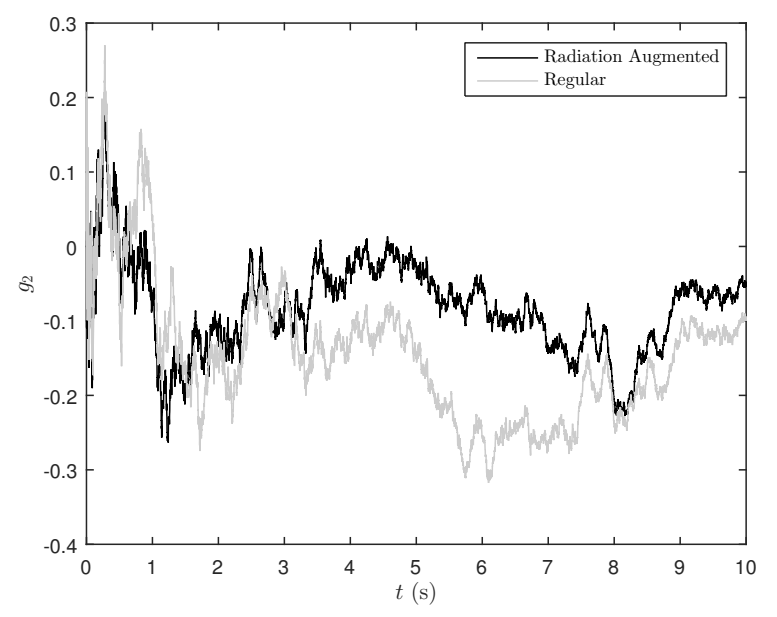

FIG. 15. Excess sample kurtosis of the colloidal particle position about the displacement due to gravity for the adaptive time step case with ${ }^{225} \mathrm{~Pa}$.

observable on timescales similar to that of the half-life, and will only be significant for short half-lives.

Results from numerical simulations have corroborated the theory. It was shown that computational time steps need to be on the order of the time between radioactive decays. Since the time between alpha decays within the colloidal particle is very short, further study with a numerical method such as molecular dynamics should be performed to understand the effect of the inertia of the fluid, transient hydrodynamic interactions between the fluid and the colloidal particle, and fluid compressibility on the motion of the colloidal particle. 


\section{ACKNOWLEDGMENTS}

The first author acknowledges the support of the Nuclear Regulatory Commission graduate fellowship program. Computing for this project was performed on the Beocat Research Cluster at Kansas State University, which is funded in part by NSF grants CNS-1006860, EPS-1006860, and EPS-0919443. CNAP Center Applications Developer Kevin Dice and Beocat Application Scientist Dave Turner provided valuable technical expertise.

${ }^{1}$ R. Brown and J. J. Bennett, The miscellaneous botanical works of Robert Brown, Vol. 1 (Published for the Ray Society by R. Hardwicke, London, 1866).

${ }^{2}$ J. L. Moran and J. D. Posner, "Phoretic self-propulsion," Annu. Rev. Fluid Mech. 49, 511-40 (2017).

${ }^{3}$ K. Kroy, D. Chakraborty, and F. Cichos, "Hot microswimmers," Eur. Phys J. Special Topics 225, 2207-2225 (2016).

${ }^{4}$ W. Zhang, Z. Liu, Y. Yang, and S. Du, "Revisiting alpha decay-based nearlight-speed particle propulsion," Applied Radiation and Isotopes 114, 1418 (2016).

${ }^{5}$ J. Chapman, "Advanced fusion reactors for space propulsion and power systems,” Tech. Rep. NF1676L-12188 (NASA Langley Research Center, Hampton, VA, 2011).

${ }^{6} \mathrm{~J}$. I. Castor, Radiation Hydrodynamics, 17th ed. (Lawrence Livermore National Laboratory, 2003)

${ }^{7}$ P. J. Coelho, "Turbulence-radiation interaction: From theory to application in numerical simulations," Journal of Heat Transfer 134 (2012).

${ }^{8}$ Advancing Nuclear Medicine Through Innovation (The National Academies Press, 2007).

${ }^{9}$ J. Elgqvist, S. Frost, J. Pouget, and P. Albertsson, "The potential and hurdles of targeted alpha therapy - clinical trials and beyond," Frontiers in Oncology 3 (2014).

${ }^{10}$ M. Hoover, D. Myers, L. J. Cash, R. Guilmette, W. Kreyling, G. Oberdorster, and R. Smith, "Radiation safety aspects of nanotechnology," Tech. Rep. 176 (National Council on Radiation Protection and Measurements, 7910 Woodmont Avenue, Suite 400 / Bethesda, MD 20814-3095, 2017).

${ }^{11}$ A. Einstein, "Über die von der molekularkinetischen theorie der wärme geforderte bewegung von in ruhenden flüssigkeiten suspendierten teilchen," Annalen der Physik 322, 549-560 (1905).

${ }^{12}$ G. E. Uhlenbeck and L. S. Ornstein, "On the theory of the brownian motion,” Physical Review 36, 823-841 (1930).

${ }^{13}$ R. Metzler, J. Jeon, A. G. Cherstvy, and E. Barkai, “Anomalous diffusion models and their properties: non-stationarity, non-ergodicity, and ageing at the centenary of single particle tracking," Phys. Chem. Chem. Phys. 16 (2014).

${ }^{14}$ P. K. Ghosh, Y. Li, G. Marchegiani, and F. Marchesoni, "Communication: Memory effects and active brownian diffusion," (2015).

${ }^{15}$ D. Debnath, P. K. Ghosh, Y. Li, F. Marchesoni, and B. Li, "Diffusion of eccentric microswimmers," Soft Matter 12, 2017-2024 (2016).

${ }^{16}$ P. K. Ghosh, V. R. Misko, F. Marchesoni, and F. Nori, "Self-propelled janus particles in a ratchet: Numerical simulations," Physical review letters 110, 268301 (2013)

${ }^{17}$ P. K. Ghosh, P. Hänggi, F. Marchesoni, and F. Nori, "Giant negative mo- bility of janus particles in a corrugated channel," Physical Review E 89, 062115 (2014).

${ }^{18}$ P. K. Ghosh, Y. Li, F. Marchesoni, and F. Nori, "Pseudochemotactic drifts of artificial microswimmers,” Physical Review E 92, 012114 (2015).

${ }^{19}$ W. B. Russel, D. A. Saville, and W. R. Schowalter, Colloidal Dispersions (Cambridge University Press, 1999)

${ }^{20} \mathrm{C}$. E. Brennen, "A review of added mass and fluid inertial forces," Tech. Rep. CR 82.010 (Naval Civil Engineering Laboratory, Port Hueneme, CA 93043, 1982).

${ }^{21}$ W. Coffey and Y. P. Kalmykov, The Langevin equation: with applications to stochastic problems in physics, chemistry and electrical engineering, Vol. 27 (World Scientific, 2012)

${ }^{22}$ E. Nelson, Dynamical theories of Brownian motion, Vol. 3 (Princeton university press, 1967).

${ }^{23} \mathrm{M}$. Abundo, "On the representation of an integrated gauss-markov process," Scientiae Mathematicae Japonicae Online e-2013, 719-723 (2013).

${ }^{24}$ J. K. Shultis and R. E. Faw, Fundamentals of Nuclear Science and Engineering, 2nd ed. (CRC Press, 2008).

${ }^{25} \mathrm{R}$. Metzler and J. Klafter, "The random walk's guide to anomalous diffusion: A fractional dynamics approach," Physics Reports 339, 1-77 (2000).

${ }^{26}$ D. J. Higham, "An algorithmic introduction to numerical simulation of stochastic differential equations," Society for Industrial and Applied Mathematics 43, 525-546 (2001).

${ }^{27} \mathrm{C}$. Yuan and X. Mao, "Stability in distribution of numerical solutions for stochastic differential equations," Stochastic Analysis and Applications 22 1133-1150 (2004).

${ }^{28}$ E. W. Weisstein, "Ball point picking," (2017), From MathWorld _ A Wolfram Web Resource. http://mathworld.wolfram.com/BallPointPicking.html.

${ }^{29}$ E. W. Weisstein, "Sphere point picking," (2017), From MathWorld _ A Wolfram Web Resource http://mathworld.wolfram.com/SpherePointPicking.html.

${ }^{30}$ G. S. Kell, "Density, thermal expansivity, and compressibility of liquid water from $0^{\circ}$ to $150^{\circ} \mathrm{c}$ : Correlations and tables for atmospheric pressure and saturation reviewed and expressed on 1968 temperature scale," Journal of Chemical and Engineering Data 20, 97-105 (1975).

${ }^{31}$ U. Onken, J. Rarey-Nies, and J. Gmehling, "The dortmund data bank: A computerized system for retrieval, correlation, and prediction of thermodynamic properties of mixtures," International Journal of Thermophysics $\mathbf{1 0}$ (1989).

${ }^{32}$ E. M. Baum, M. C. Ernesti, H. D. Knox, T. R. Miller, and A. M. Watson, Nuclides and Isotopes Chart of the Nuclides, 17th ed. (Bechtel Marine Propulsion Corporation, 2009).

${ }^{33}$ S. C. Wu, "Nuclear data sheets for a $=214$," Nuclear Data Sheets 110, 681 748 (2009).

${ }^{34}$ A. K. Jain and B. Singh, "Nuclear data sheets for a $=218$," Nuclear Data Sheets 107, 1027-1074 (2006).

${ }^{35}$ A. K. Jain, S. Singh, S. Kumar, and J. K. Tuli, "Nuclear data sheets for a = 221," Nuclear Data Sheets 108, 883-922 (2007).

${ }^{36}$ J. F. Ziegler, M. D. Ziegler, and J. P. Biersack, "Srim - the stopping and range of ions in matter (2010)," Nuclear Instruments and Methods in Physics Research B 268, 1818-1823 (2010).

${ }^{37}$ K. V. Mardia, "Measures of multivariate skewness and kurtosis with applications," Biometrika 57, 519-530 (1970). 

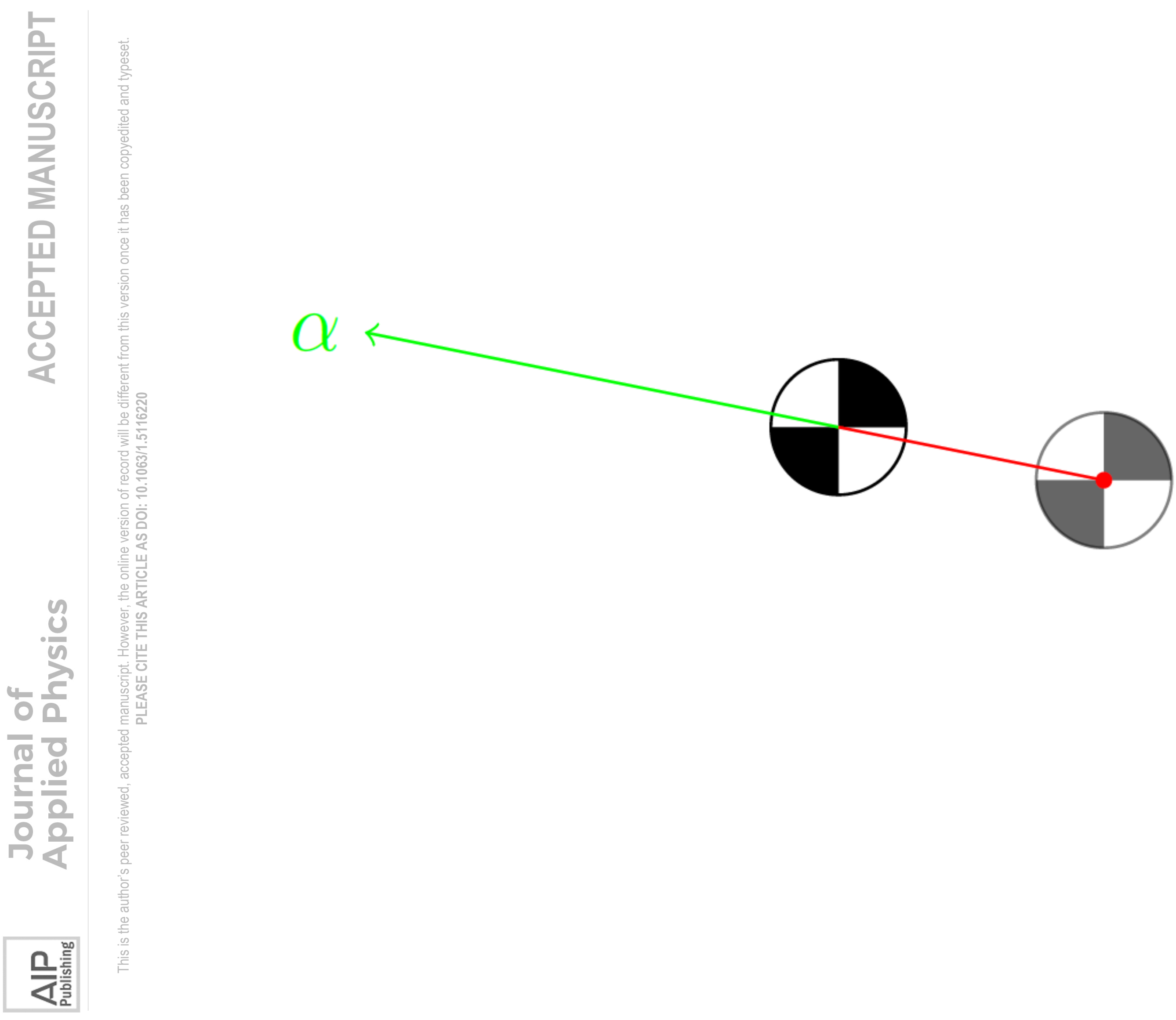


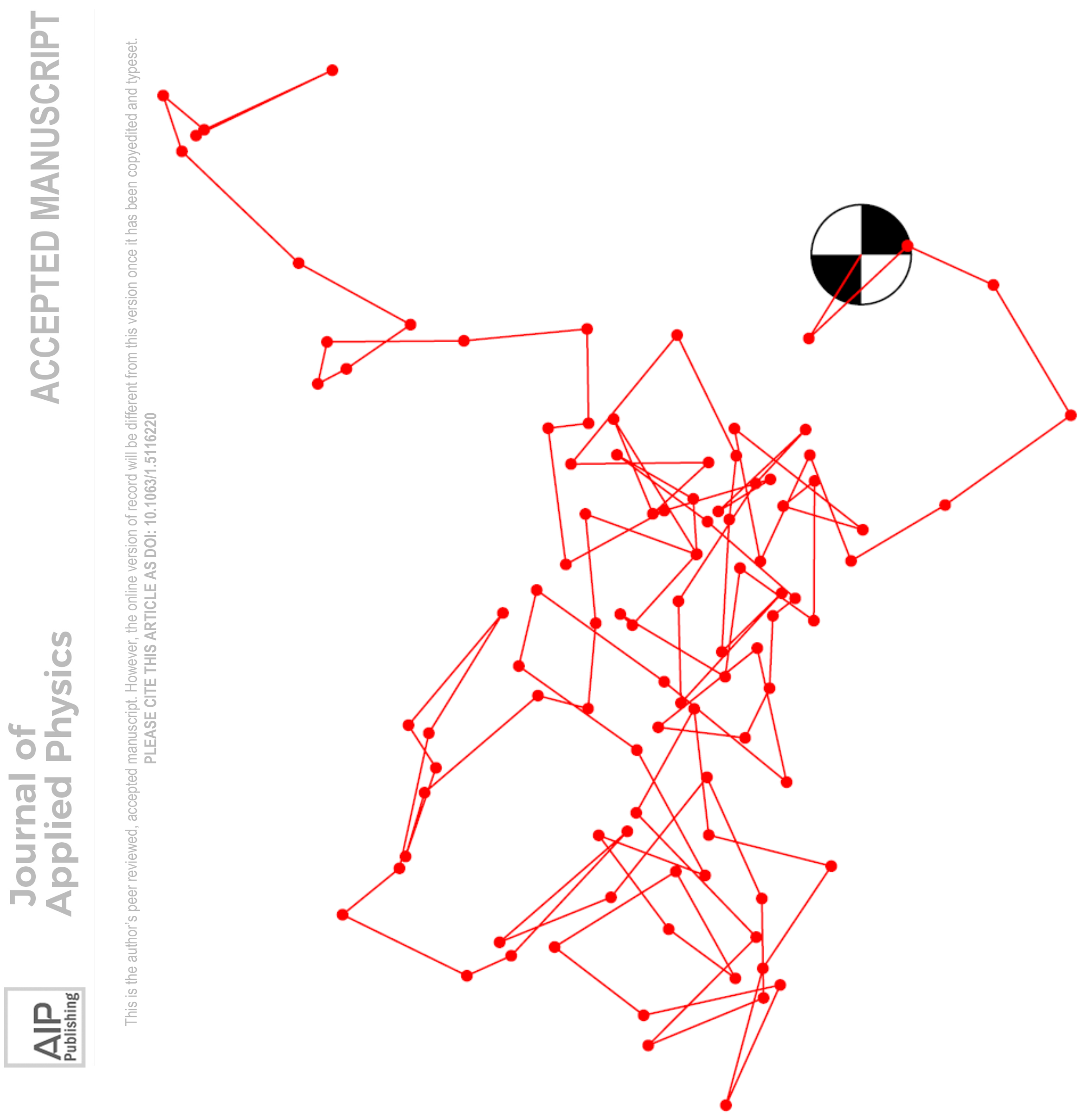




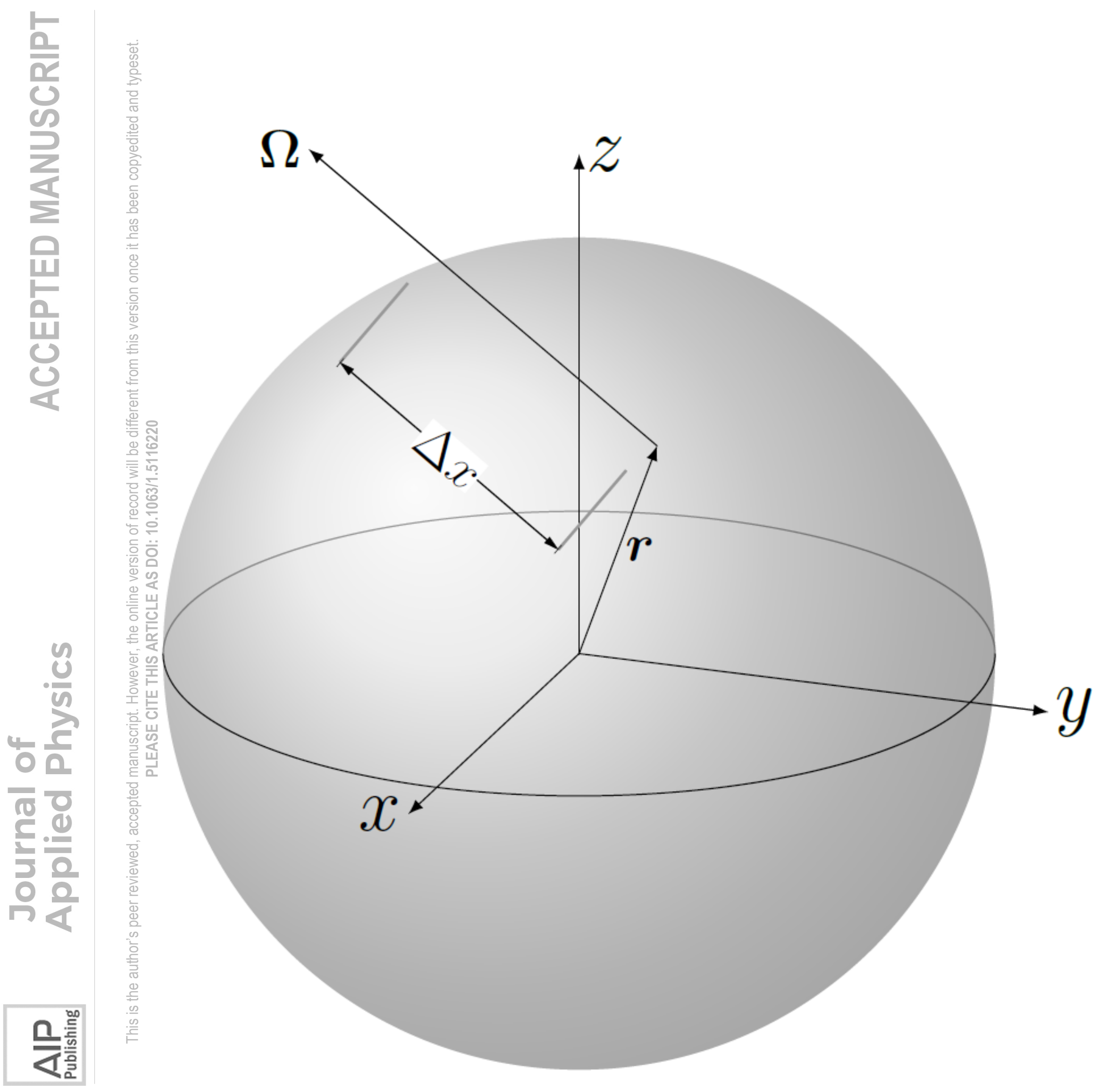




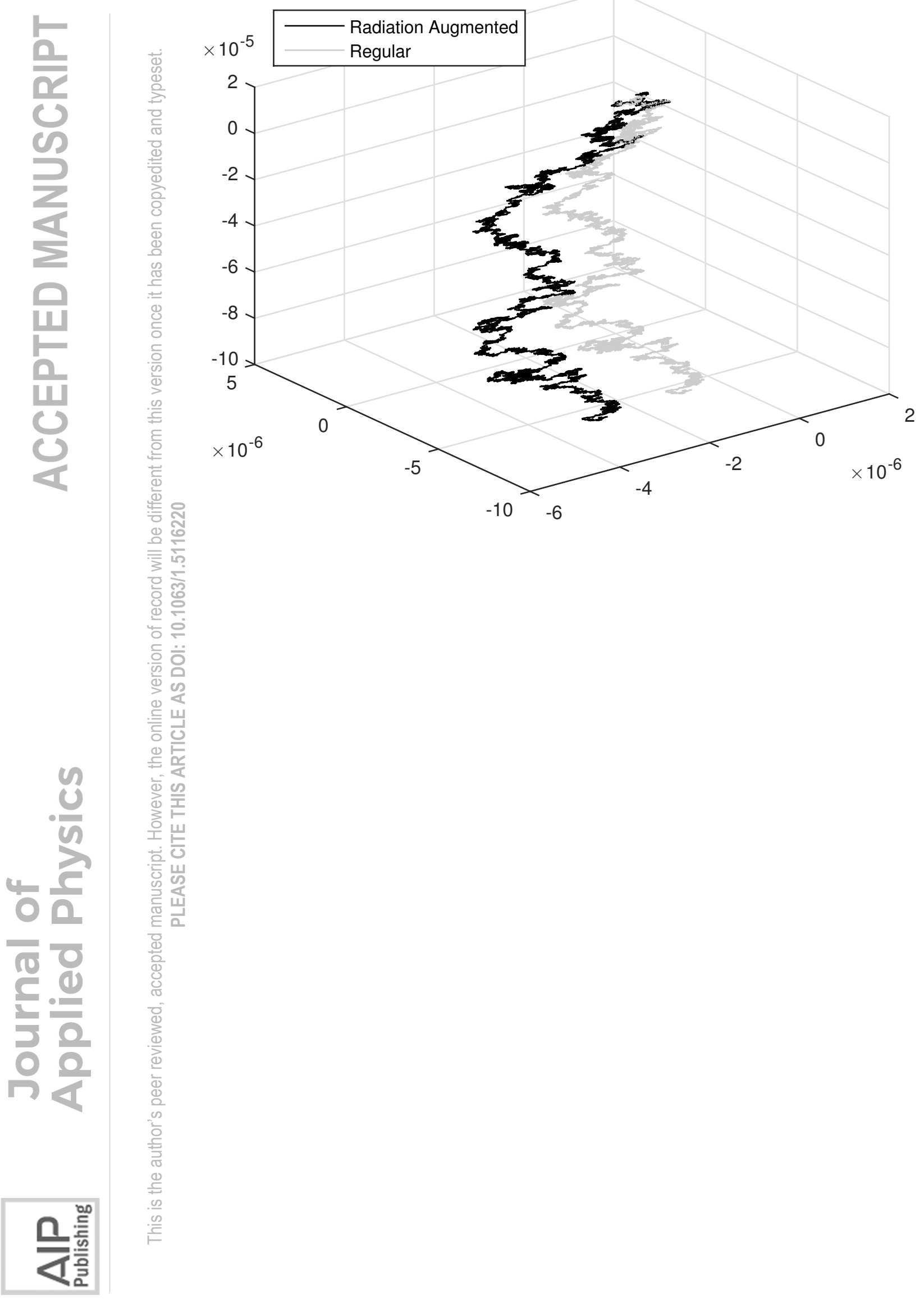




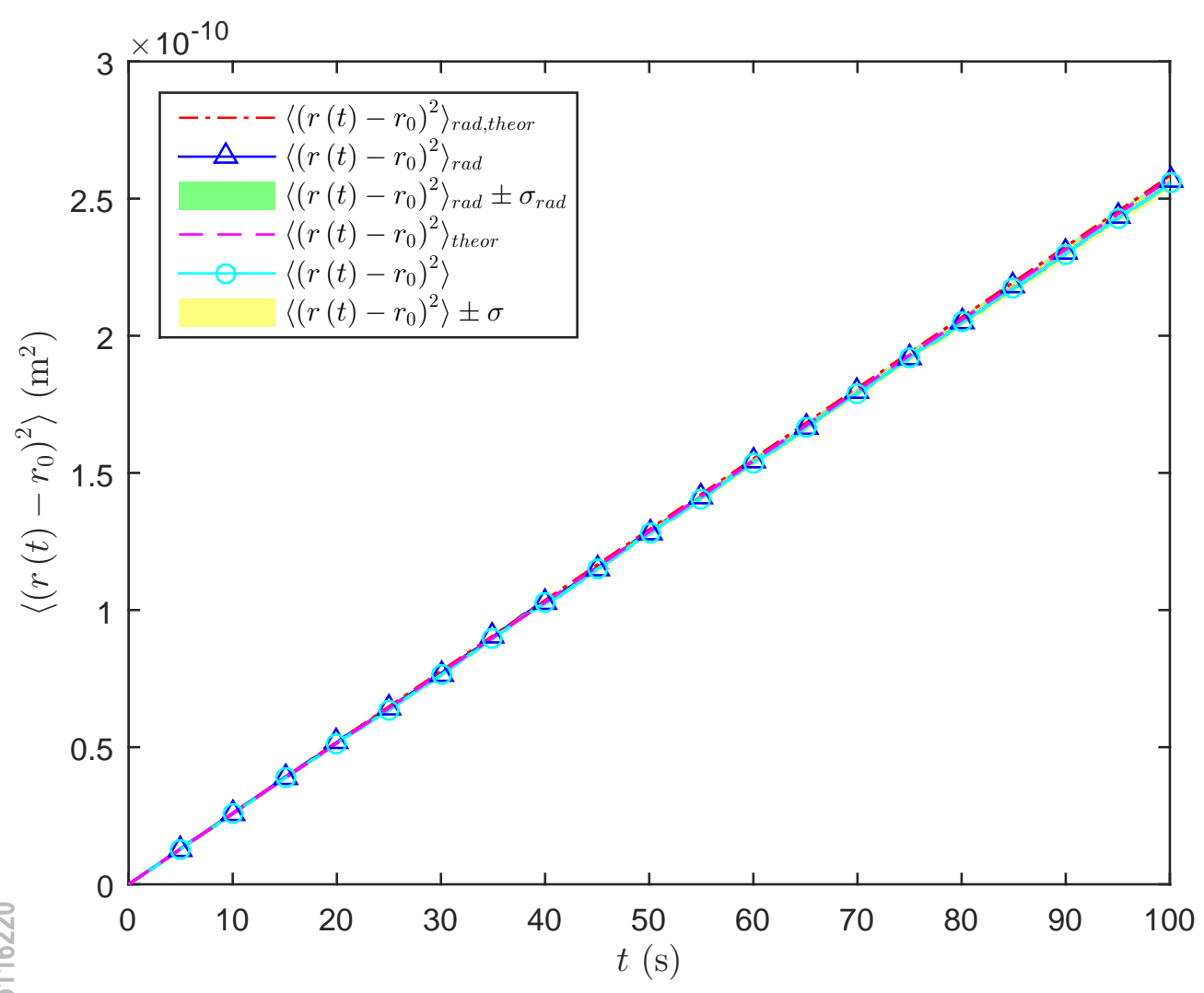




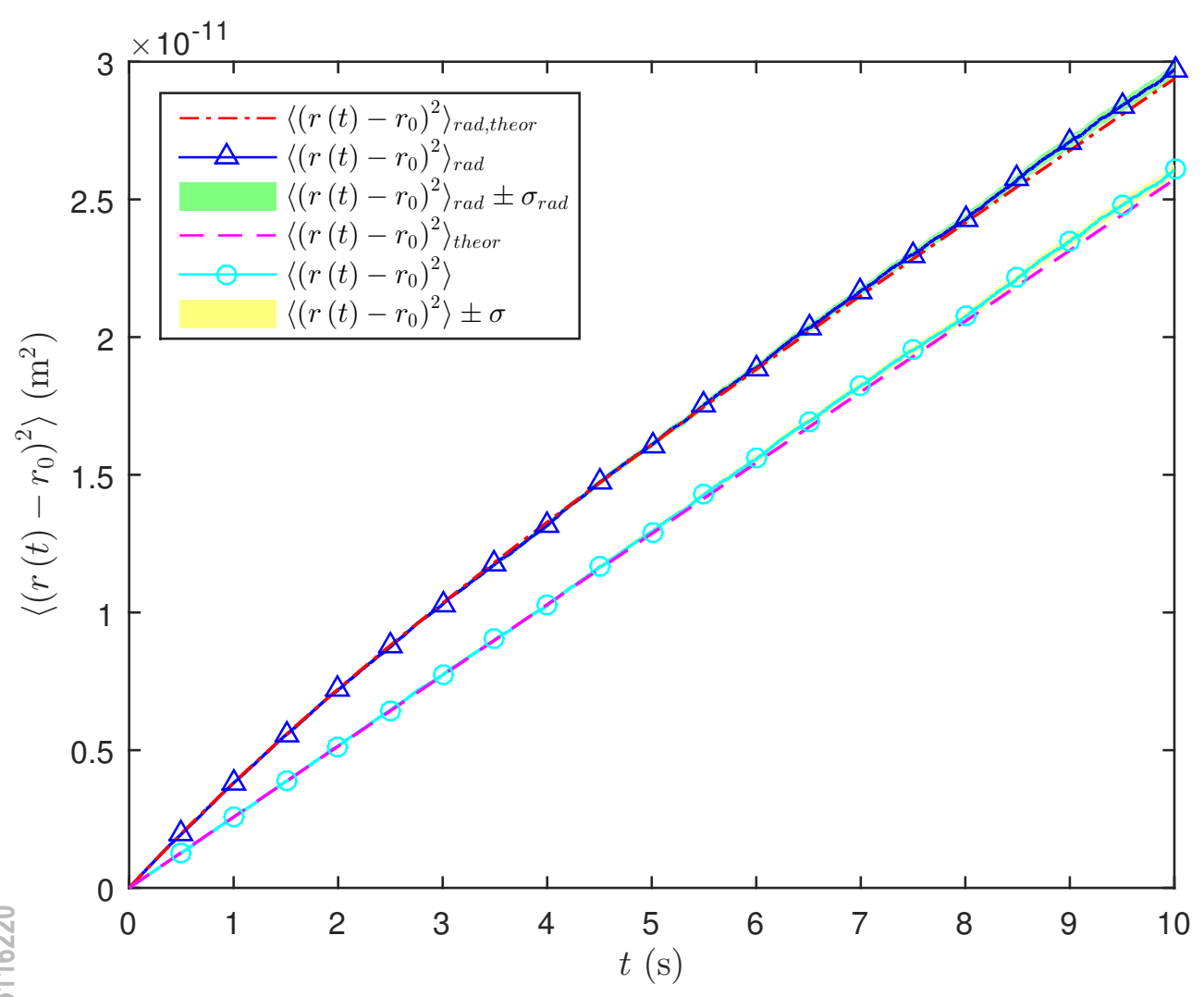




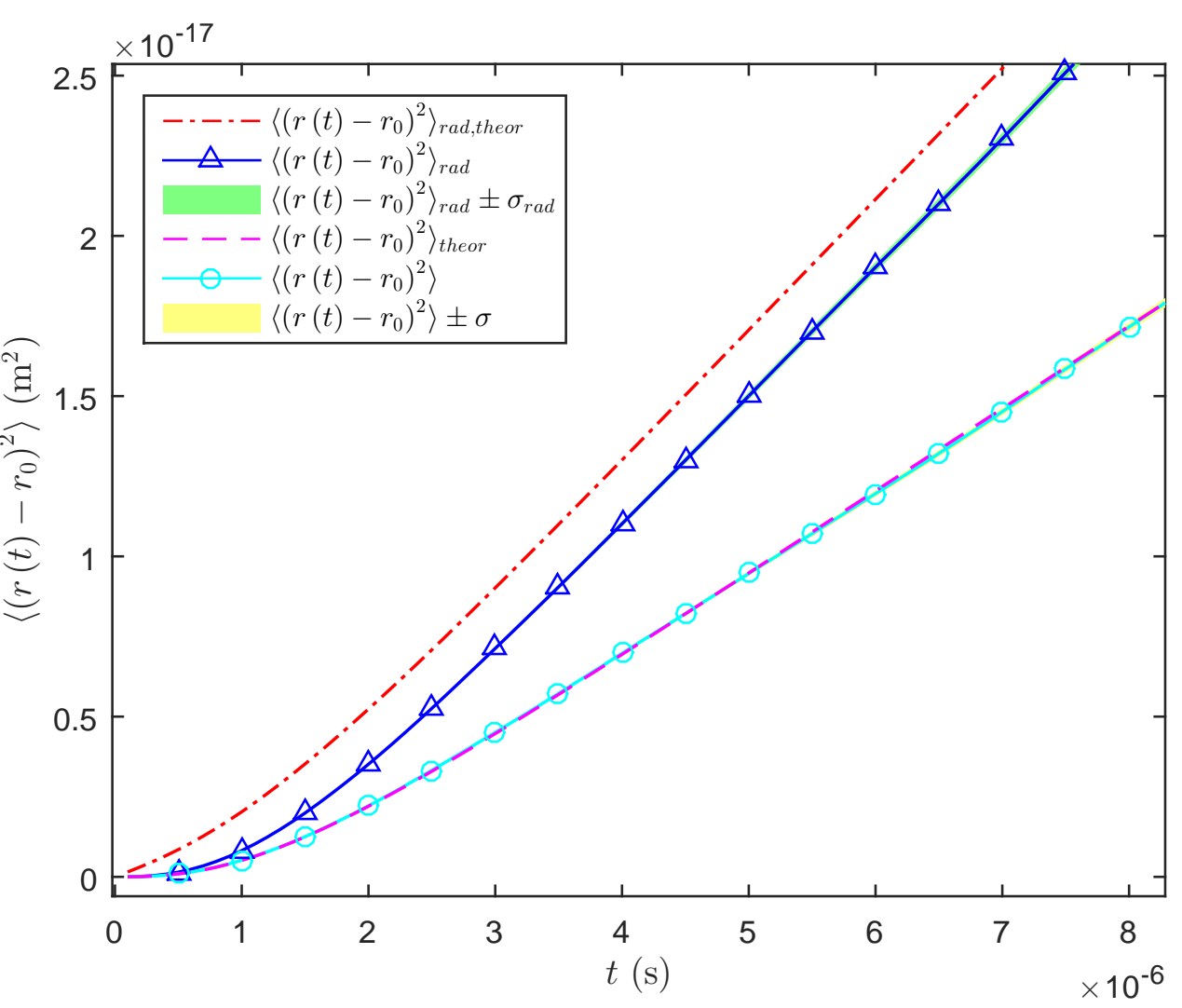




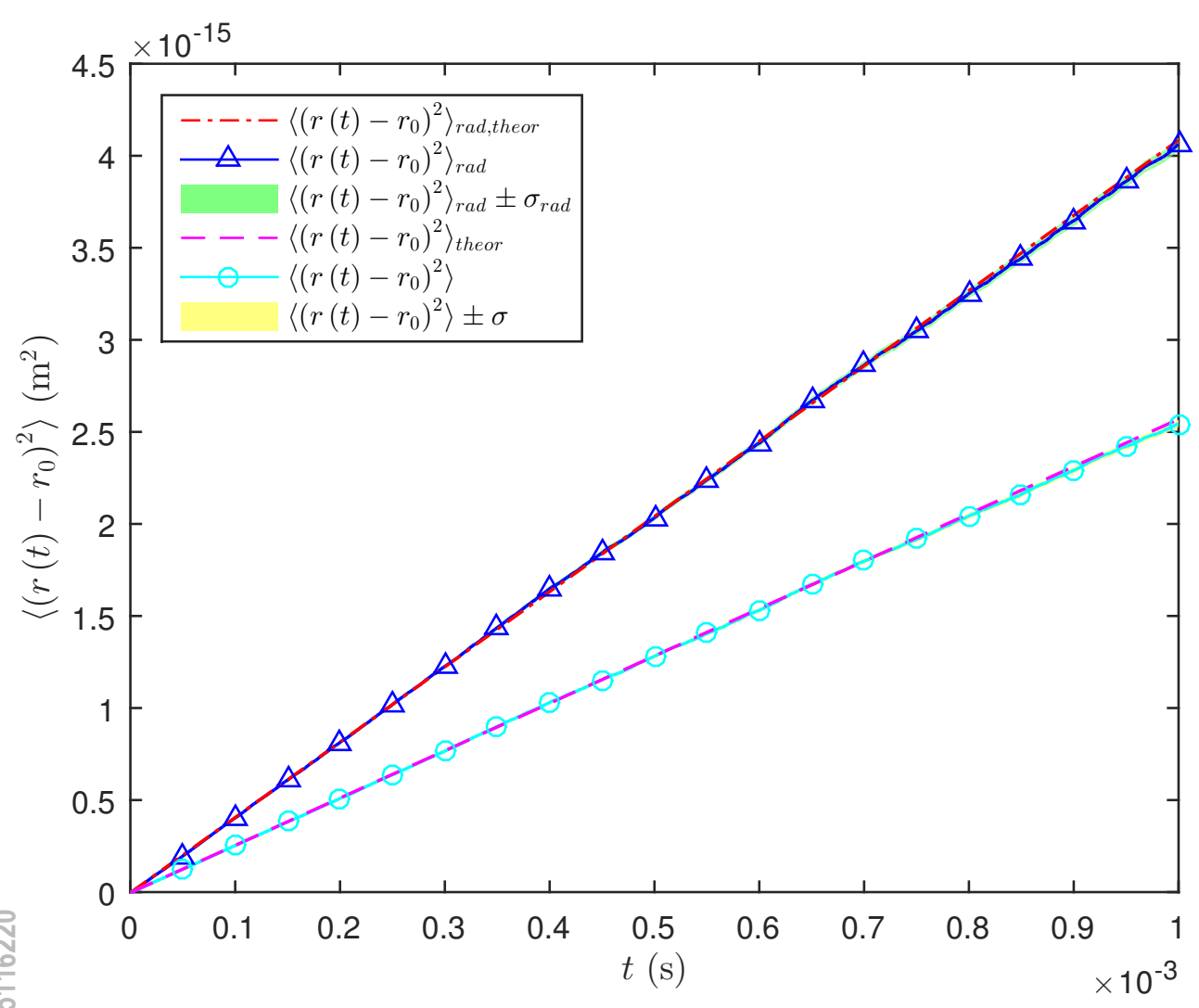




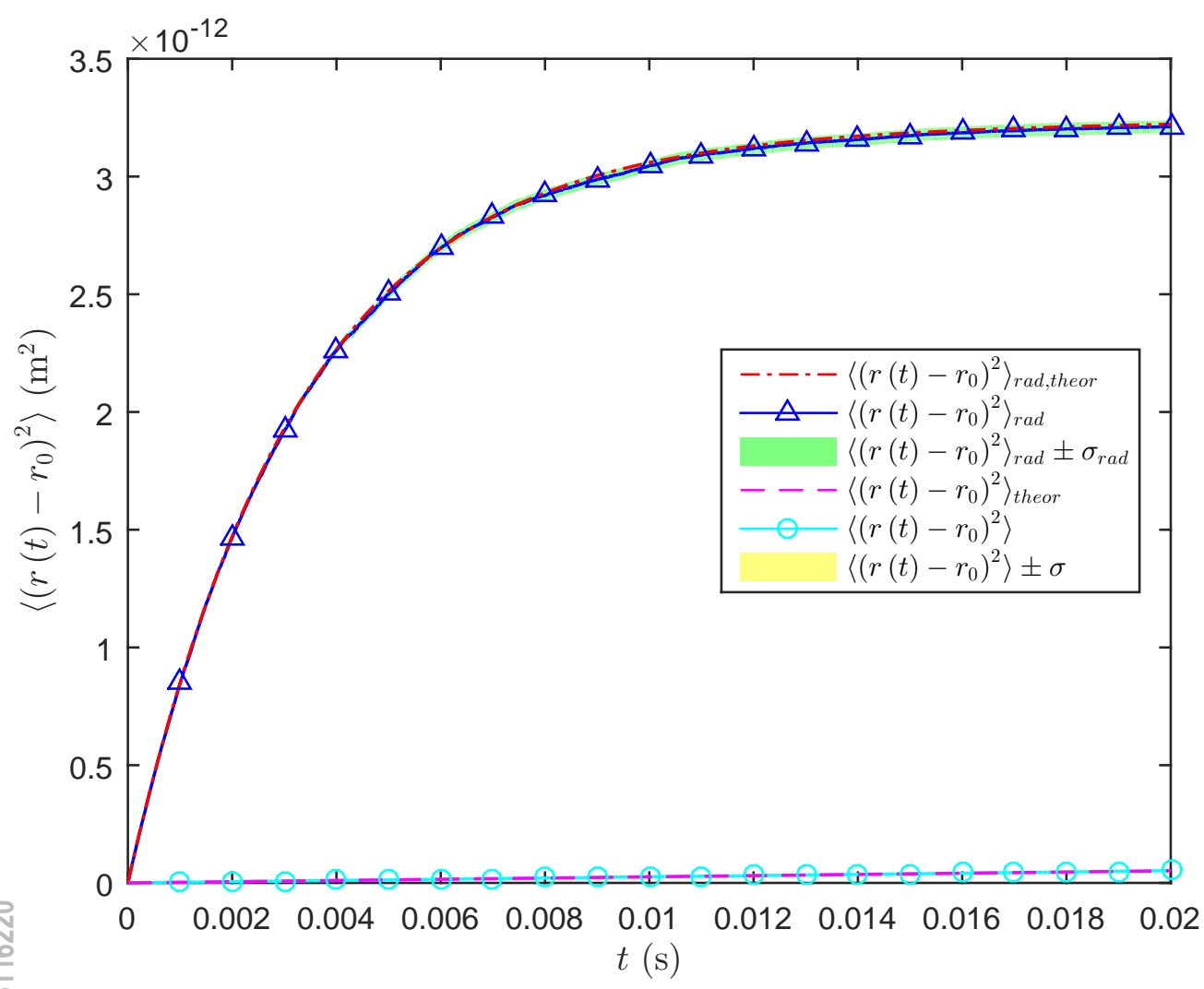




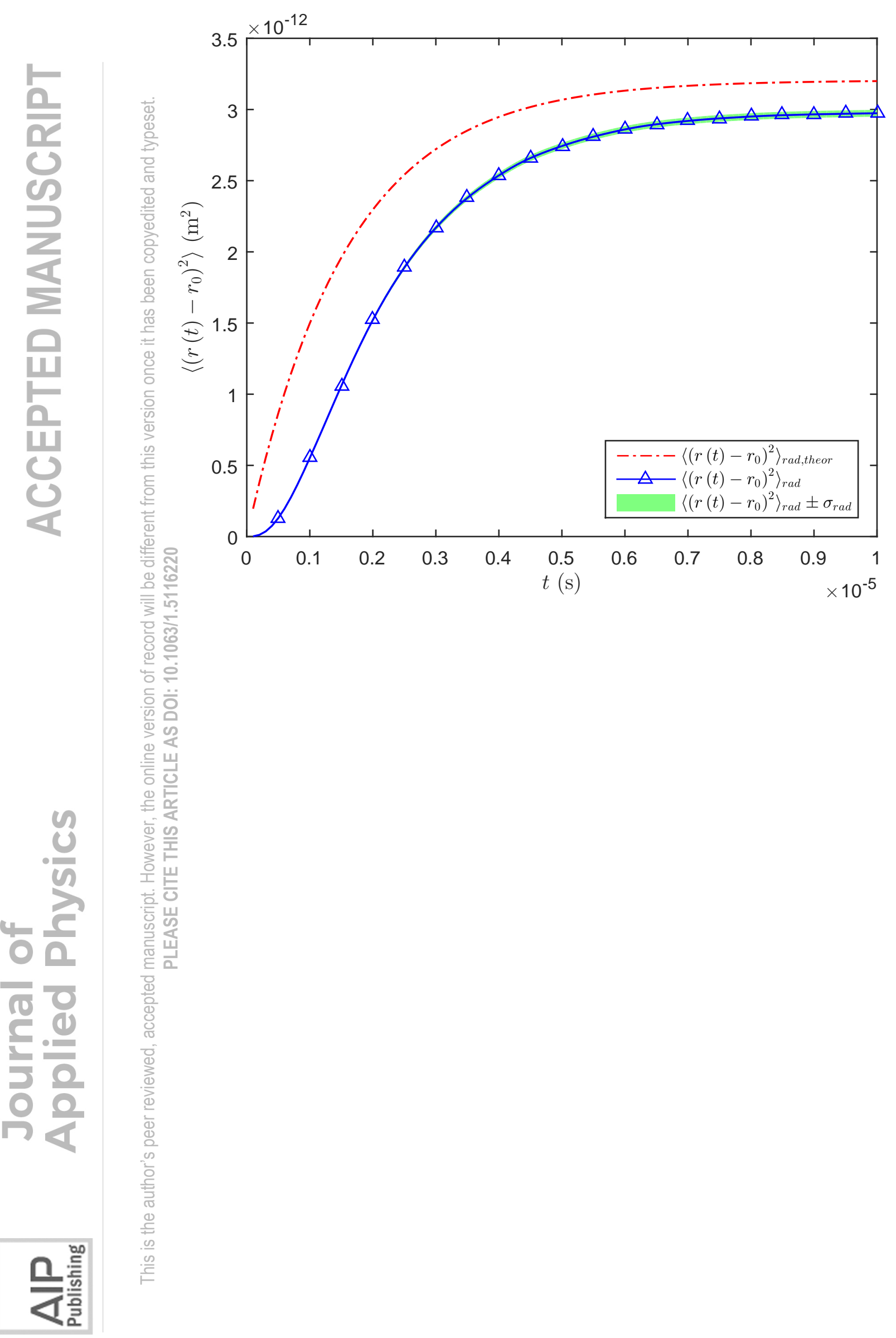




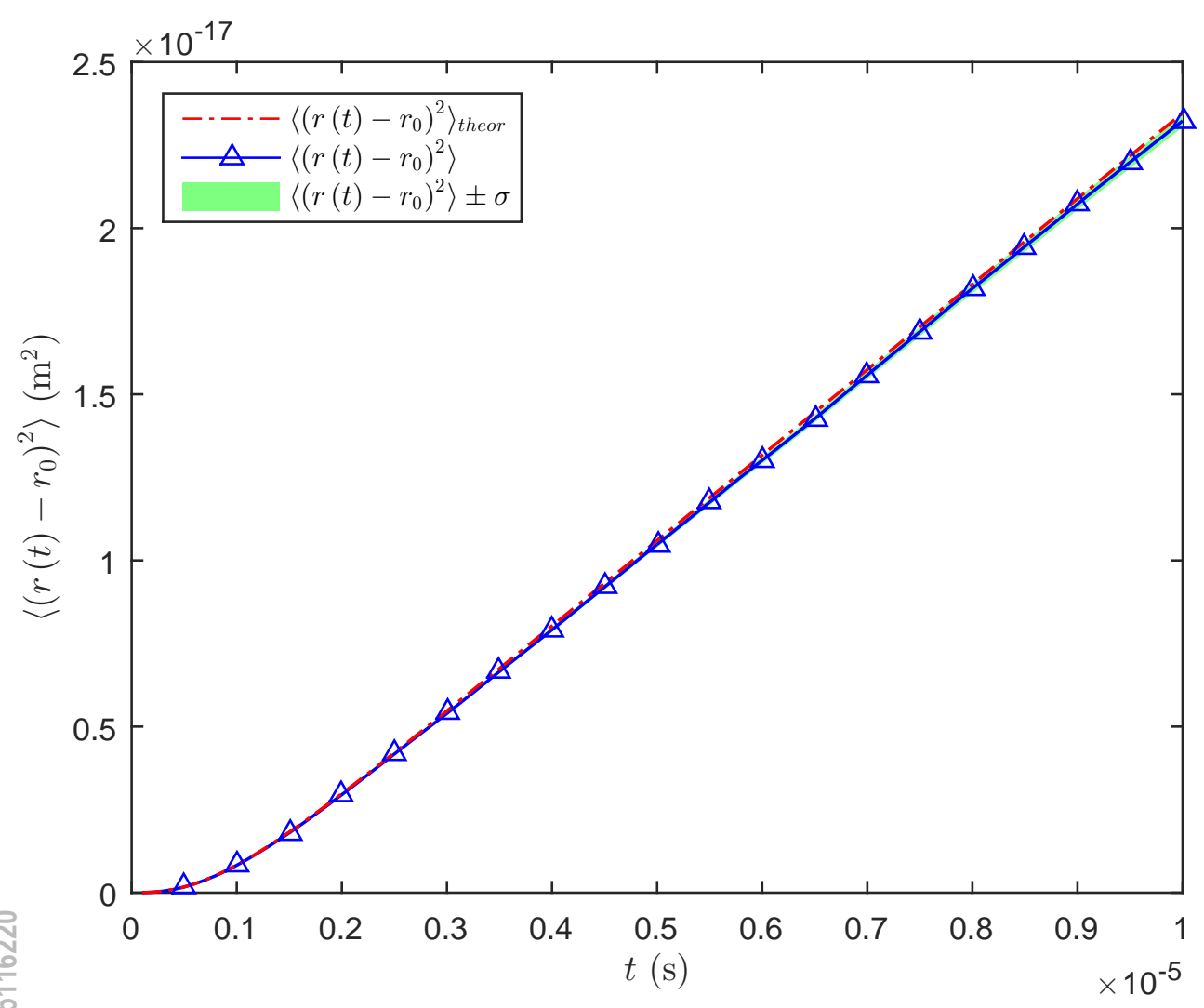




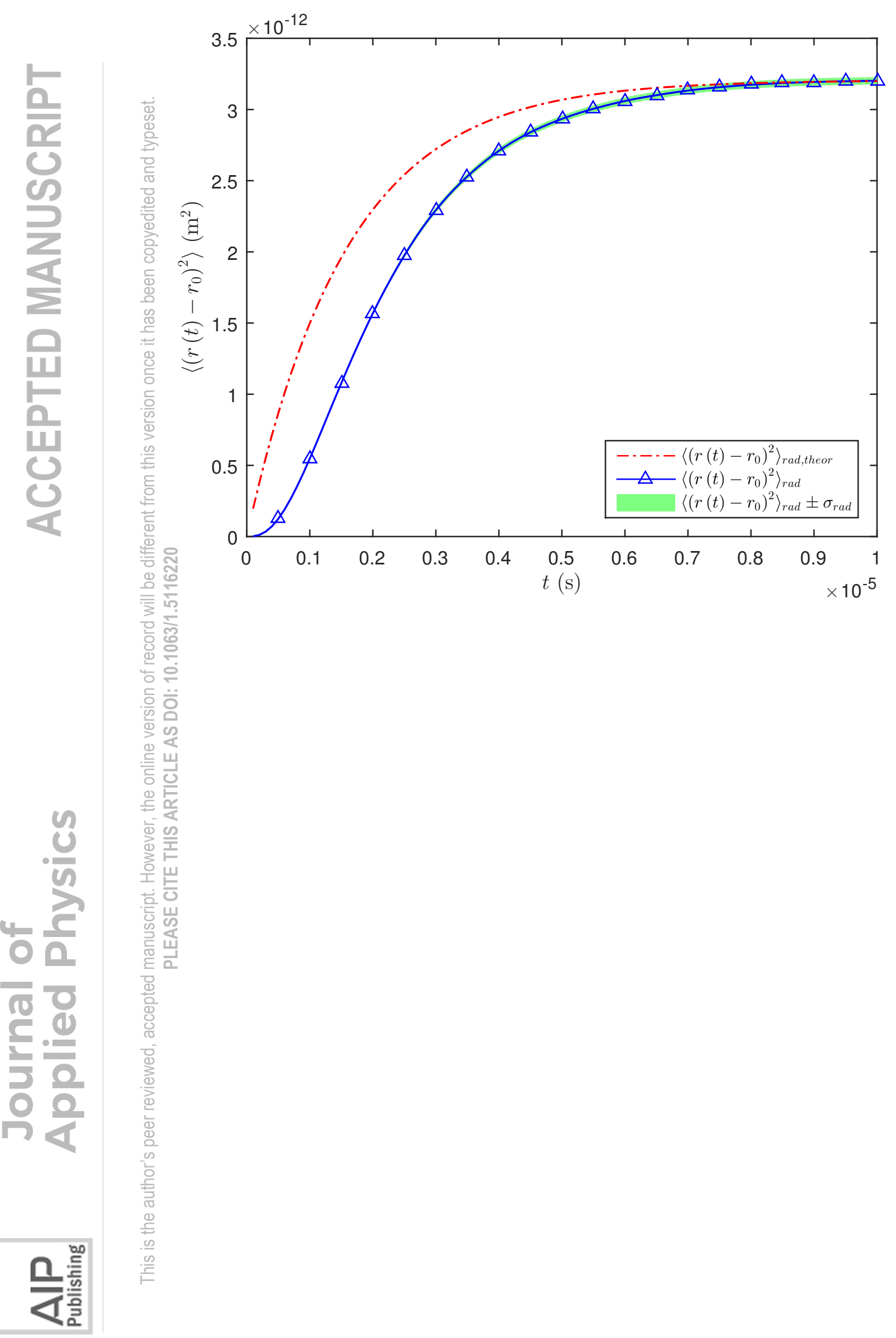




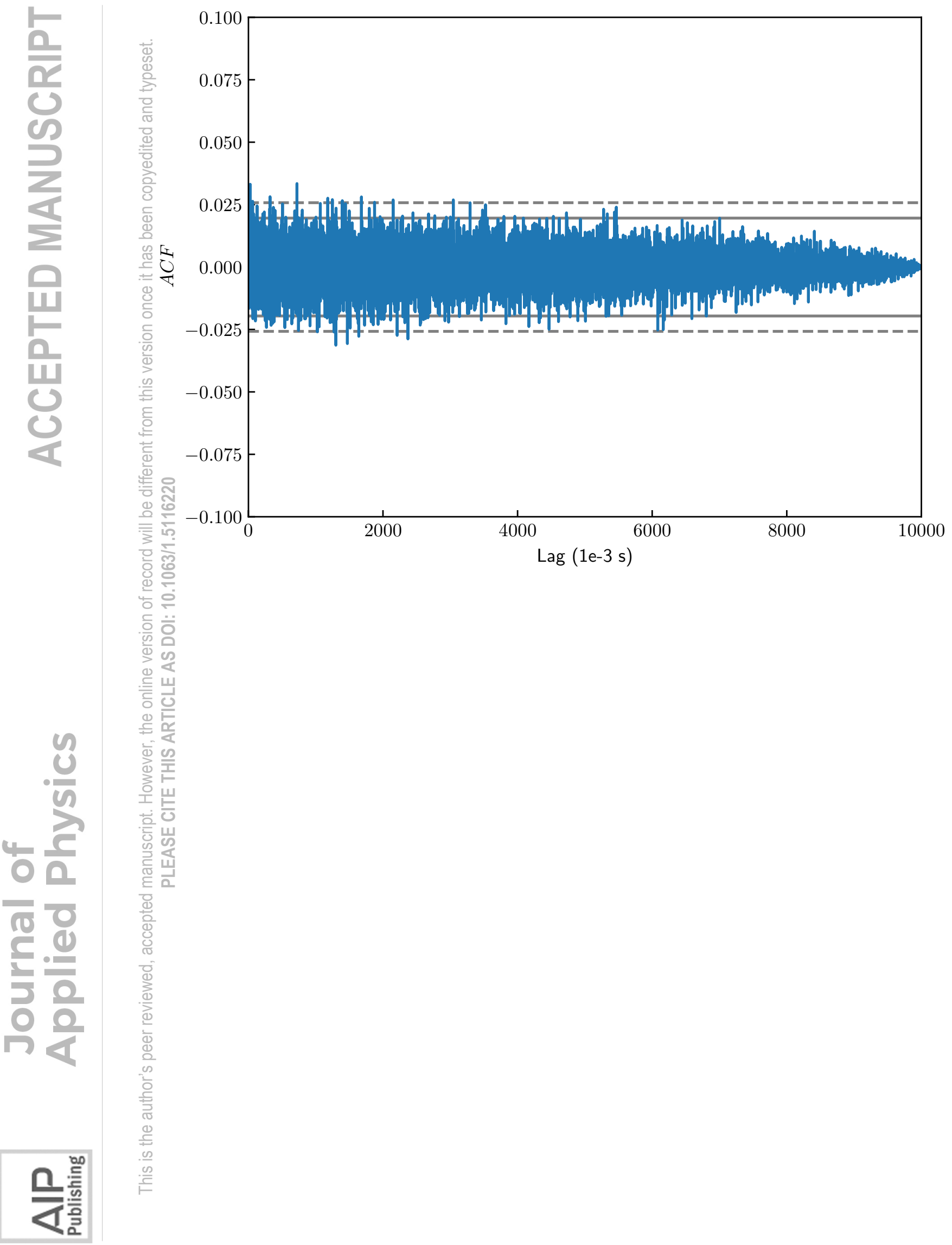




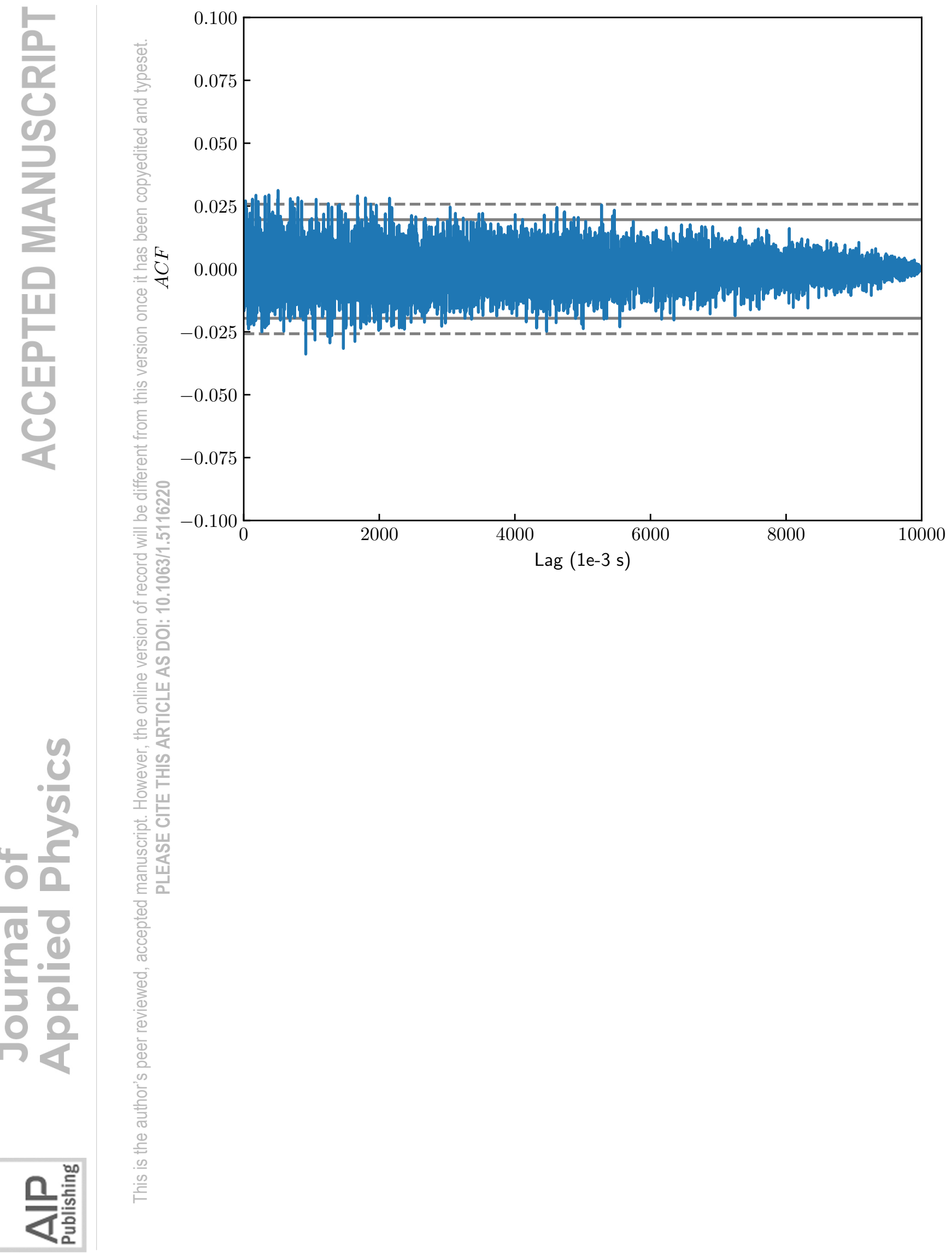




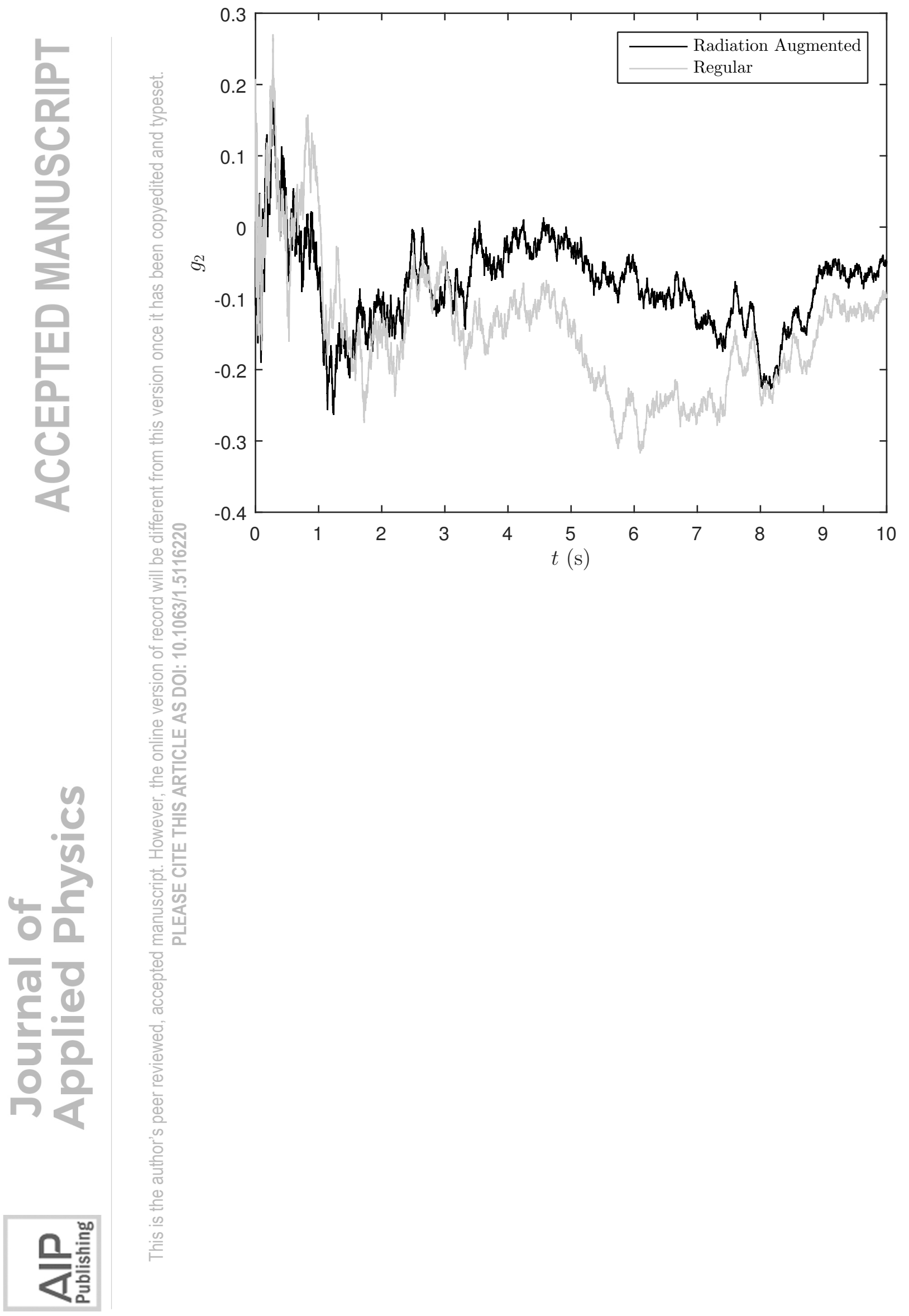

